\title{
Model-Based Adaptive Control of Transfemoral Prostheses: Theory, Simulation, and Experiments
}

\author{
Vahid Azimi $^{\circledR}$, Tony Shu, Huihua Zhao, Rachel Gehlhar, Dan Simon ${ }^{\circledR}$, and Aaron D. Ames ${ }^{\circledR}$
}

\begin{abstract}
This paper presents and experimentally implements three different adaptive and robust adaptive controllers as the first steps toward using model-based controllers for transfemoral prostheses. The goal of this paper is to translate these control methods to the robotic domain, from bipedal robotic walking to prosthesis walking, including a rigorous stability analysis. The human/prosthesis system is first modeled as a two-domain hybrid asymmetric system. An optimization problem is formulated to obtain a stable human-like gait. The proposed controllers are then developed for the combined human/prosthesis model and the optimized reference gait. The stability of all three controllers is proven using the Lyapunov stability theorem, ensuring convergence to the desired gait. The proposed controllers are first verified on a bipedal walking robot as a hybrid human/prosthesis model in simulation. They are then experimentally tested on a treadmill with an able-bodied subject using third iteration of AMBER Prosthetic (AMPRO3), a custom self-contained powered transfemoral prosthesis. Finally, outdoor tests are carried out using AMPRO3 with three test subjects walking on level ground, uphill slopes, and downhill slopes at slope angles of $3^{\circ}$ and $8^{\circ}$, to demonstrate walking in different real-world environments.
\end{abstract}

Index Terms-Adaptive and robust adaptive control, hybrid system, transfemoral prosthesis, walking biped.

\section{INTRODUCTION}

$\mathbf{T}$ HE NUMBER of transfemoral amputees in the United States is at around 222000 [1]. Persons with amputation can utilize prosthetic legs to re-establish typical activities of daily living. There are three general types of prosthetic legs: 1) passive; 2) active; and 3) semi-active [2], [3]. Among them, active prostheses enable amputees to walk with a more natural gait compared with the other types while simultaneously allowing the users to walk more efficiently at different

Manuscript received November 25, 2018; accepted January 20, 2019. This work was supported by NSF under Award NRI-1526519 and Grant 1344954. This paper was recommended by Associate Editor Z. Li. (Corresponding author: Vahid Azimi.)

V. Azimi is with the School of Electrical and Computer Engineering, Georgia Institute of Technology, Atlanta, GA 30308 USA (e-mail: vahid.azimi@gatech.edu).

T. Shu is with MIT Media Lab, Massachusetts Institute of Technology, Cambridge, MA 02139 USA.

H. Zhao is with Driving, Toyota Research Institute, San Francisco, CA 95125 USA.

R. Gehlhar and A. D. Ames are with the Department of Mechanical and Civil Engineering, California Institute of Technology, Pasadena, CA 91125 USA.

D. Simon is with the Department of Electrical Engineering and Computer Science, Cleveland State University, Cleveland, OH 44115 USA.

Color versions of one or more of the figures in this paper are available online at http://ieeexplore.ieee.org.

Digital Object Identifier 10.1109/TSMC.2019.2896193
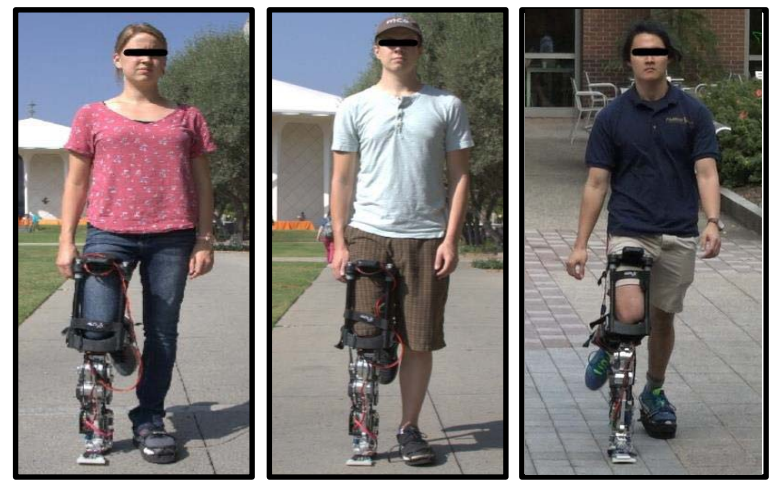

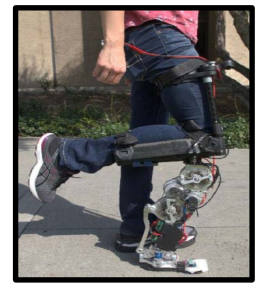

Subject \#1

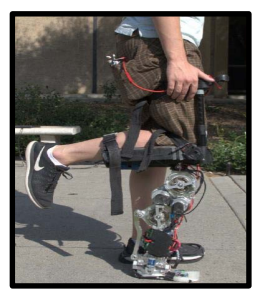

Subject \#2

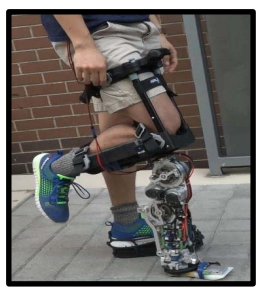

Subject \#3
Fig. 1. Outdoor test with three healthy test subjects wearing the powered transfemoral prosthesis, AMPRO3.

speeds with less fatigue [4]. Motivated by the large number of above-knee amputations and advantages of active prostheses, researchers have recently concentrated on design and control of powered prostheses [5], [6].

In this paper, three model-based controllers are formulated for powered transfemoral prostheses as a means of addressing limitations of model-free control approaches, such as variable impedance (VI) and proportional-derivative (PD) controllers. The objective is to guarantee convergence/boundedness of system solutions in the presence of modeling error and disturbances through a rigorous systematic stability analysis. All proposed controllers are first implemented on a 5-link planar walking biped model in simulation and then experimentally tested using third iteration of AMBER Prosthetic (AMPRO3), which is shown in Fig. 1. Simulations show that the proposed controllers effectively meet performance requirements such as tracking and robustness to force/obstacle disturbances. Treadmill test results show that all proposed controllers provide human-like walking and good prosthesis knee tracking. A comprehensive comparison is then performed during the treadmill test to show superiority of the proposed modelbased controllers over the VI and PD controllers. The outdoor 
test is performed with three test subjects as shown in Fig. 1 walking on level ground, uphill slopes, and downhill slopes at slope angles of $3^{\circ}$ and $8^{\circ}$. Outdoor test results show that the proposed controllers provide convergence of the system trajectories to a stable limiting periodic orbit, resulting in stable walking using AMPRO3 on all tested terrains.

\section{A. Background}

At present, model independent VI control is the most common approach for controlling active prosthetic legs, especially in the context of impedance control [7], [8]. However, impedance control suffers several shortcomings: tedious impedance parameter tuning (unique to each specific amputee subject), difficulties in determining subphase thresholds, lack of feedback, and passiveness [7], [9]. To address some of the limitations of ordinary impedance control, a prosthetic impedance controller combining a control Lyapunov function with model independent quadratic programs was recently developed in [10] and [11]. In those papers, the proposed controller was tested on the planar 5-link bipedal robot advanced mechanical bipedal experimental robot (AMBER) and AMBER Prosthetic (AMPRO), a self-contained powered transfemoral prosthesis, both in simulation and experiment. The authors achieved better tracking performance and improved energy efficiency compared to VI control. To address the tuning problem of the VI controllers, automatic tuning approaches have been recently used for control of a powered prosthetic leg [12]. However, since the above-mentioned controllers were designed in a model-free fashion, the formal stability analysis of the controllers was not provided.

The PD control paradigm is another popular approach in robotics applications. With this paradigm, gravitational effects are unknown, and this type of controller yields steady state tracking error. Since perfect knowledge of the system dynamics is rarely available, the VI and PD controllers are designed independently of model information, involving only the tracking error in their structures (called model-free controllers). As a consequence, these approaches do not take into account model information and are not able to include model uncertainty in the stability analysis. In other words, although model-free controllers are able to provide reasonable control performance, a formal proof is hard to derive. Thus, the aforementioned controllers lack a formal guarantee of stability and robustness in the presence of system uncertainties, unmodeled dynamics, and disturbances. However, these influences may degrade the performance of the closed-loop system or even lead to instability. A rigorous systematic stability analysis is required to guarantee convergence/boundedness of the system in the presence of unknown model uncertainties and external disturbances. Through this stability analysis, convergence of error trajectories is formally guaranteed in the presence of unknown system information about user and prosthesis dynamics. This analysis can be provided only by using model-based controllers to guarantee the control objectives for different users, different prostheses, and in the presence of unknown bounded model errors and disturbances.
There have been a few attempts to analyze the stability of VI control in robotics applications. In [13], a tank-based approach to impedance control with variable stiffness is proposed based on the total energy of the manipulator. In that approach, the stiffness profile is state-dependent and modified online. Its performance is heavily dependent on the initial and threshold levels of energy in the tank. These shortcomings make this method sensitive to perturbations and prone to instability. As an improvement to the state-dependent tank-based approach, stability considerations for VI control are proposed in [14]. That work provides a stability condition for varying stiffness and damping that is state independent and can be found offline. However, that method requires model knowledge, dynamic decoupling, and measurement of external effort in interaction tasks.

Several results of model-based controllers have recently been attained on robot manipulators, prostheses, humanoid robots, and servo systems [15]-[19]. In [17], an active disturbance rejection adaptive controller (ADC) was designed for a class of nonlinear systems with modeling uncertainty. Both parametric uncertainties and uncertain nonlinearities were estimated and then compensated in a feedforward way. The motion control of a motor-driven robot manipulator was investigated to verify the tracking performance of the control strategy. In [18], an adaptive integral robust controller was designed for a class of uncertain nonlinear systems. To ensure robustness against disturbances, the controller gain was updated online without prior bound knowledge of the disturbances. An electromechanical servo system driven by a motor was considered to verify the effectiveness of the proposed controller. In [19], four robust model reference adaptive impedance observer/controller combinations were designed for ground reaction force (GRF) estimation-based control of a prosthesis and a legged robot model. These approaches were designed to control the system while compensating for the GRF effects, parametric uncertainties, and unmodeled dynamics.

\section{B. Contributions}

Motivated by the aforementioned shortcomings, this paper is the first step toward designing model-based adaptive and robust adaptive control for prosthetic walking. Stability and robustness of the proposed controllers are formally guaranteed in the presence of model uncertainties and disturbances such as unforeseen forces and obstacles.

In our prior work [20], two different robust controllers: 1) robust sliding mode controller (RSC) and 2) robust passivity-based controller (RPC), were designed for AMPRO3. This present paper includes the following important contributions beyond previous work.

1) Formulation of adaptive and robust $\mathrm{ADCs}$ for bipedal robotic walking (human/prosthesis system) with formal stability and robustness analyses.

2) Evaluation of tracking performance and force/obstacle disturbance robustness on the walking biped platform as shown in Fig. 2(a). 


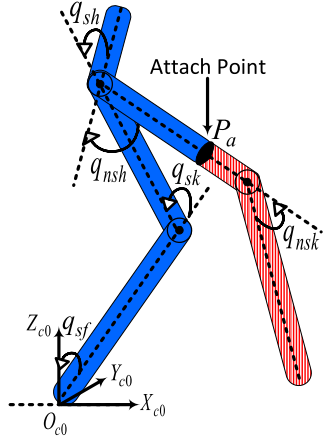

(a)

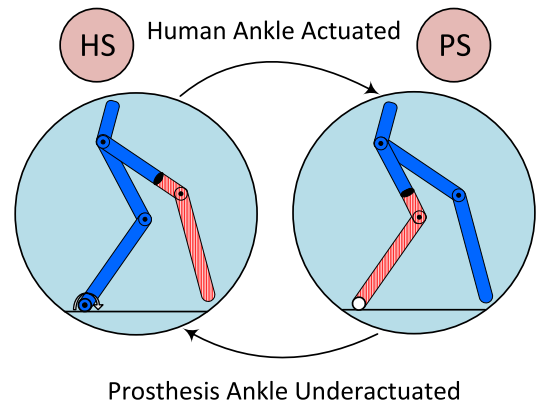

(b)
Fig. 2. (a) 5-link planar model with point prosthetic foot, where the red cross-hatched part represents the prosthesis. (b) Two-domain hybrid asymmetric human-prosthesis system with actuated human ankle and underactuated prosthesis ankle.

3) Experimental verification with treadmill walking by an able-bodied subject of the proposed controllers of the powered transfemoral prosthesis AMPRO3 as shown in Fig. 1.

4) Experimental demonstration with treadmill walking of the superiority of the proposed model-based controllers compared to the VI and PD controllers with regard to tracking performance and torque optimality.

5) Experimental outdoor demonstration of AMPRO3 with the proposed controllers by three test subjects on level ground, uphill slopes, and downhill slopes, for slope angles of $3^{\circ}$ and $8^{\circ}$ as shown in Fig. 1.

In this paper, the prosthesis is first modeled with the prismatic-prismatic-revolute-revolute (PPRR) joint structure. The hybrid human/prosthesis system is then modeled using a point prosthetic foot 5-link planar model comprised of one torso, two thighs, and two shanks. An optimization problem is formulated to provide optimal and stable human-like gait. For the human/prosthesis system and the generated gait, three different model-based controllers are designed: the pure ADC, the robust sliding mode ADC (RSAC), and the adaptive integral controller (AIC). The stability of all proposed controllers is proven using the Lyapunov stability theorem for continuous dynamics of the prosthesis system.

In this paper, relying on the learning and adaptation nature of ADCs, the ADC is designed to estimate unknown prosthetic leg parameters and user mass in order to achieve good tracking performance. To take one step further toward enhancing tracking performance, the AIC is designed using a timevarying sliding surface exploiting the integral of position error. However, the ADC and AIC only investigate parametric uncertainties while ignoring unmodeled dynamics and disturbances. The aforementioned advantages of adaptive control along with the presence of both disturbances and nonparametric uncertainties motivates the blending of ADC and RSC to construct the RSAC. The RSAC can provide robustness to all parametric and nonparametric uncertainties, and provide convergence of error trajectories of the system to a boundary layer. This robustness is maintained while the unknown parametric uncertainty of the system is identified by an adaptation mechanism. The proposed RSAC can achieve the same control objectives as [17]-[19], but with a simpler and more straightforward design procedure.

Model-free neural-network (NN)-based ADCs can also be employed for robust adaptive control. These controllers can be used to eliminate the need for the knowledge of the dynamic model structure [21]-[24]. However, such controllers require a more complicated control structure and incur more computational cost compared to model-based ADCs. Because the modeling of the prosthesis device (Section II-B) is a straightforward task, there is no need to choose the NN-based $\mathrm{ADC}$, and a simpler control structure such as the model-based controllers proposed here are preferred.

The goal is to translate the proposed methods to the robotic domain, taking inspiration from bipedal locomotion. The proposed controllers in this paper are first verified in simulation on a walking biped platform and then compared with RSC and RPC with respect to robustness and tracking performance. Simulation results illustrate that all three proposed systems have good tracking performance and robustness, qualitatively emulating human-like walking. All three proposed controllers are then experimentally verified on AMPRO3 with the human test subject walking for $2.5 \mathrm{~min}$ at a treadmill speed of $2 \mathrm{mph}$, achieving good tracking and reasonable prosthesis knee torque values. Treadmill test results also show that the proposed model-based controllers outperform the VI and PD controllers with regard to tracking performance and torque requirements. In addition to the treadmill test, outdoor tests are performed using AMPRO3 with three test subjects (as shown in Fig. 1) walking on different outdoor terrains: level ground, uphill, and downhill at slope angles of $3^{\circ}$ and $8^{\circ}$. Outdoor tests reveal that the proposed controllers provide stable periodic walking not only on level ground but also on uneven surfaces. Our experiments and simulations can be seen in a video [25].

This paper is organized as follows. Section II describes the combined human/prosthesis system and presents the problem statement. Section III presents the proposed controller structures along with their stability analyses. Section IV presents simulation results for tracking performance and robustness to disturbances. Section V presents experimental results on AMPRO3. Section VI presents discussion, concluding remarks, and future work.

\section{Human/Prosthesis SySTEM AND PROBLEM STATEMENT}

A human/prosthesis system can be modeled as a walking biped. It is, therefore, reasonable to adopt bipedal robots as a platform to test the prosthesis controllers. In this section, modeling and control of a 5-link planar walking biped (one torso, two thighs, and two shanks) shown in Fig. 2(a) is first studied. The prosthetic device is then modeled using a PPRR joint structure for the prosthesis control formulation. Finally, problem statement and proposed control architecture are explained. 


\section{A. Modeling and Control}

A walking biped (i.e., a system with impulse effects) can be modeled as a hybrid system consisting of a sequence of continuous (leg swinging forward) and discrete (impact at foot strike) events. The human/prosthesis walking structure can be defined with configuration space $Q_{R}$ in local coordinates $q_{c}=\left(q_{\mathrm{sf}}, q_{\mathrm{sk}}, q_{\mathrm{sh}}, q_{\mathrm{nsh}}, q_{\mathrm{nsk}}\right)^{T}$ with world frame $O_{\mathrm{co}}=\left\{x_{\mathrm{co}}, y_{\mathrm{co}}, z_{\mathrm{co}}\right\}$ as illustrated in Fig. 2(a). The formal definition of hybrid systems can be found in [26] and [27]. As shown in Fig. 2(a), $q_{\mathrm{sf}}$ is the angle of the stance foot, $q_{\mathrm{sk}}$ is the angle of the stance knee, $q_{\mathrm{sh}}$ is the angle of the stance hip, $q_{\text {nsh }}$ is the angle of the nonstance hip, and $q_{\text {nsk }}$ is the angle of the nonstance knee. Using the Euler-Lagrange formula [28], the equations of motion of the bipedal continuous dynamics are given as

$$
M_{c}\left(q_{c}\right) \ddot{q}_{c}+C_{c}\left(q_{c}, \dot{q}_{c}\right) \dot{q}_{c}+g_{c}\left(q_{c}\right)=B u_{c}
$$

where $M_{c}\left(q_{c}\right) \in \mathfrak{R}^{5 \times 5}$ is the inertia matrix; $C_{c}\left(q_{c}, \dot{q}_{c}\right) \in \mathfrak{R}^{5 \times 5}$ is the Coriolis and centripetal matrix; $g_{c}\left(q_{c}\right) \in \mathfrak{R}^{5 \times 1}$ is the gravity vector; $B \in \mathfrak{R}^{5 \times 5}$ is the torque map with underactuated prosthesis side and actuated human side; and $u_{c} \in \mathfrak{R}^{5 \times 1}$ is the vector of torque inputs.

When the nonstance foot hits the ground, angular velocities of the human-prosthesis bipedal model change upon impact while the stance and nonstance legs are switched. The discrete dynamics of the biped can therefore be considered as the impact dynamics, which are derived based on the holonomic constraints applied for the subsequent domain [27]. To consider impulse effects of the combined system right before and after impact, a reset map with the assumption of perfect plastic impact [29] is presented as [30]

$$
\Delta_{r}\left(q_{c}, \dot{q}_{c}\right)=\left[\begin{array}{c}
\Delta_{q_{c}} q_{c} \\
\left.\Delta_{\dot{q}_{c}} q_{c}\right) \dot{q}_{c}
\end{array}\right]
$$

where $\Delta_{q_{c}}$ relabels and switches the stance and nonstance leg at impact and $\Delta_{\dot{q}_{c}}$ gives the velocity change due to impact.

To emulate human-like walking, actual combined system outputs $y_{a}$ must converge to desired human outputs $y_{d}$. The desired human outputs $y_{d}(t, \xi)$ (a function of time and parameter set $\xi$ ) for walking and running can be defined as the time solution to a linear mass-spring-damper system by the canonical walking function (CWF) [31]

$$
y_{d}(t, \xi)=e^{-\xi_{1} t}\left(\xi_{2} \cos \left(\xi_{3} t\right)+\xi_{4} \sin \left(\xi_{3} t\right)\right)+\xi_{5}
$$

where the relation between the CWF of (3) and the solution to a linear mass-spring-damper system reveals that $\xi_{1}=\xi_{d} \omega_{n}$ with $\xi_{d}$ as the damping ratio and $\omega_{n}$ as the natural frequency; $\xi_{5}=g$ is the gravity and $\xi_{3}=\omega_{n}$; and $\xi_{2}$ and $\xi_{4}$ are determined by the initial conditions of the system. Note that the number of the parameters is $N_{\xi}=5$.

Therefore, convergence of the actual human outputs $y_{a}\left(q_{c}, \dot{q}_{c}\right)$ (function of the biped's states) to the desired human outputs $y_{d}(t, \xi)$ of (3) implies convergence of their difference to zero. In other words, the human-inspired controller can be applied to drive the following outputs to zero:

$$
y\left(q_{c}, \dot{q}_{c}, \xi\right)=y_{a}\left(q_{c}, \dot{q}_{c}\right)-y_{d}(t, \xi)
$$

where $y\left(q_{c}, \dot{q}_{c}, \xi\right)$ is comprised of relative degree one and relative degree two virtual constraints. For the point prosthetic foot 5-link planar model in this paper, the actual outputs $y_{a}$ are comprised of forward hip velocity $\left(\nu_{\mathrm{hip}_{x}}\right)$, stance knee angle (ska), nonstance knee angle (nska), nonstance leg slope (nsls), and torso angle [32]. Note that forward hip velocity is the relative degree one output, which is a function of both biped position and velocity $\left(y_{a 1}\left(q_{c}, \dot{q}_{c}\right)\right)$, and the other outputs are considered relative degree two outputs, which are only a function of biped configuration variables $\left(y_{a 2}\left(q_{c}\right)\right)$. Thus, the number of the relative degree one and two outputs, respectively are $N_{y_{a 1}}=1$ and $N_{y_{a 2}}=4$. Also, note that the parameters of all of the outputs is $\xi=\left(v_{\text {hip }_{x}}, \xi_{\text {ska }}, \xi_{\text {nska }}, \xi_{\text {nsls }}, \xi_{\text {ta }}\right) \in \Re^{\left(N_{\xi} \times N_{y_{a 2}}\right)+1}$, so the total number of parameters is $N_{\xi_{t}}=\left(N_{\xi} \times N_{y_{a 2}}\right)+1$.

Since forward hip velocity of the biped, which is the first actual output, is roughly constant during walking, its forward hip position can be approximated as $p_{\text {hip }_{x}}=v_{\text {hip }_{x}} t$ [33]. The constant forward hip velocity is an important attribute of human walking, and $t=p_{\text {hip }_{x}} / v_{\text {hip }_{x}}$. For the sake of timeinvariance of the control structure, $t$ in the time-based desired human output $y_{d}(t, \xi)$ of (3) can be replaced by the following parameterized time [26]:

$$
\tau\left(q_{c}\right)=\frac{p_{\text {hip }_{x}}\left(q_{c}\right)-p_{\text {hip }_{x}}\left(q_{c}^{0}\right)}{v_{\text {hip }_{x}}}
$$

where $p_{\text {hip } x}$ is the forward hip position and $p_{\text {hip }}\left(q_{c}^{0}\right)$ denotes its initial value. Using this parametrization of time, the humaninspired outputs of (4) can be rewritten as

$$
y\left(q_{c}, \dot{q}_{c}, \xi\right)=\left(\begin{array}{c}
y_{1}\left(q_{c}, \dot{q}_{c}, \xi\right) \\
y_{2}\left(q_{c}, \xi\right)
\end{array}\right)=\left(\begin{array}{c}
y_{a 1}\left(q_{c}, \dot{q}_{c}\right)-v_{\mathrm{hip}_{x}} \\
y_{a 2}\left(q_{c}\right)-y_{d}\left(\tau\left(q_{c}\right), \xi\right)
\end{array}\right)
$$

where $v_{\text {hip }}$ and $y_{d}\left(\tau\left(q_{c}\right), \xi\right)$ are state-based desired outputs for the relative degree one and relative degree two human outputs, respectively.

Although the feedback linearization human-inspired controller drives the aforementioned human-inspired outputs to zero and provides exponential stability of the continuous dynamics, it is also essential to keep these outputs zero upon impact. While the relative degree one output (forward velocity) of a human is relatively constant during walking, relaxing the hip velocity constraint is desirable when impact occurs. Therefore, the following partial zero dynamics (PZD) surface is only considered on the relative degree two output $y_{2}\left(q_{c}, \xi\right)$ :

$$
\mathcal{P} Z_{\xi}=\left\{\left(q_{c}, \dot{q}_{c}\right) \in Q_{R}: y_{2}\left(q_{c}, \xi\right)=0, \dot{y}_{2}\left(q_{c}, \xi\right)=0\right\} .
$$

To guarantee invariance of the reset map $\Delta_{r}$ of the PZD of each domain, the partial hybrid zero dynamics (PHZD) are introduced as [30], [34]

$$
\text { PHZD : } \triangle_{r}\left(S_{r} \cap \mathcal{P} \mathcal{Z}_{\xi}\right)=\mathcal{P} \mathcal{Z}_{\xi}
$$

(PHZD)

where $S_{r}$ denotes the switching surface of the biped model for transitions to the next domain.

Now, the goal is to find the set $\xi \in \Re^{\left(N_{\xi} \times N_{y_{a 2}}\right)+1}$ using an optimization problem subject to both PHZD and other physical constraints (foot clearance in addition to position and velocity 


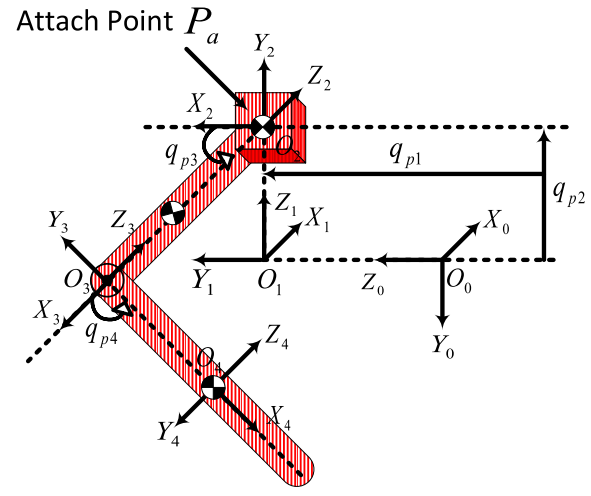

Fig. 3. Transfemoral prosthesis model.

joint limits), providing an optimal and stable human-like gait

$$
\begin{aligned}
\xi^{*}=\underset{\xi \in \Re\left(N_{\xi} \times N_{y_{a 2}}\right)+1}{\operatorname{argmin}} \operatorname{COST}(\xi) \\
\text { s.t. PHZD \& Physical Constraints. }
\end{aligned}
$$

COST is a human data-based cost function, which is the leastsquares-fit error between human walking data obtained from inertial measurement units (IMUs) and the CWF [31]. This function is defined as

$$
\begin{aligned}
\operatorname{CosT}(\xi)= & \sum_{i=1}^{N_{y_{2}}} \sum_{j=1}^{N_{p}}\left[y_{a 2_{i}}\left(q_{c}[j]\right)-y_{d_{i}}\left(t_{i}[j], \xi_{i}\right)\right]^{2} \\
& +\sum_{j=1}^{N_{p}}\left[p_{\text {hip }_{x}}\left(q_{c}[j]\right)-\left(v_{\text {hip }_{x}} t[j]+p_{\text {hip }_{x}}\left(q_{c}[0]\right)\right)\right]^{2}
\end{aligned}
$$

where $t[j]$ is the discrete time and $N_{p}$ is the number of discrete time points.

The first part of COST calculates the set $\left(\xi_{\text {ska }}, \xi_{\text {nska }}, \xi_{\text {nals }}\right.$, and $\xi_{\text {ta }}$ ) by least squares fitting of the CWF and the human data of the relative degree two outputs. The second part determines the constant $v_{\text {hip }_{x}}$ by minimizing the difference between the actual forward hip position and the linearized one from (5). Thus, by solving this optimization problem, the set $\xi^{*}$ can be found so that $\operatorname{COST}\left(\xi^{*}\right) \leq \delta$ for some small positive constant $\delta$. This implies that when the outputs of the walking biped follow the desired human outputs $y_{d}\left(t, \xi^{*}\right)$, the human/prosthesis system acts like a linear mass-spring-damper system. In this paper, the optimization problem of (8) is performed by the MATLAB function fmincon to find the set $\xi \in \mathfrak{R}^{21}$.

It should be pointed out that when the parameter set $\xi$ is optimized subject to PHZD constraints, tracking invariance of the relative degree two outputs is guaranteed even at impact. This provides smooth transitions between stance and nonstance phases. With desired human-inspired outputs from the optimization problem of (8), desired joint angles and angular velocities of the combined system $\left(q_{c}^{d}\right)$ are calculated via inverse projection from the PHZD surface using the PHZD reconstruction procedure [27].

\section{B. Prosthesis Model}

The prosthetic device [red portion in Fig. 2(a)] can be modeled using a PPRR joint structure as illustrated in Fig. 3. The equations of motion of the prosthetic leg are

$$
M_{p}\left(q_{p}\right) \ddot{q}_{p}+C_{p}\left(q_{p}, \dot{q}_{p}\right) \dot{q}_{p}+g_{p}\left(q_{p}\right)=u_{p_{i}}+d_{p}(t)
$$

where $q_{p}=\left(q_{p 1}, q_{p 2}, q_{p 3}, q_{p 4}\right)^{T}, q_{p 1}$, and $q_{p 2}$ are the horizontal and vertical displacements of the attach point $P_{a}$ respectively, and $q_{p 3}$ and $q_{p 4}$ are thigh and knee angles respectively; $M_{p}\left(q_{p}\right) \in \mathfrak{R}^{4 \times 4}, C_{p}\left(q_{p}, \dot{q}_{p}\right) \in \mathfrak{R}^{4 \times 4}$, and $g_{p}\left(q_{p}\right) \in \mathfrak{R}^{4 \times 1}$ are the inertia matrix, Coriolis and centripetal matrix, and gravity vector respectively; $u_{p_{i}} \in \mathfrak{R}^{4 \times 1}$ is the prosthesis control signal generated by the $i$ th controller $(i=1,2,3)$ presented in Section III, where $u_{p_{i}}$ is comprised of horizontal and vertical forces at the hip and active control torques at the thigh and knee; and $d_{p}(t) \in \mathfrak{R}^{4 \times 1}$ is a time varying disturbance. Further details about this model can be found in [20].

The following properties of the model of (10) are used when developing the proposed algorithms.

Property 1: The inertia matrix $M_{p}\left(q_{p}\right)$ is symmetric, positive definite, and uniformly bounded.

Property 2: The matrix $\dot{M}_{p}\left(q_{p}\right)-2 C_{p}\left(q_{p}, \dot{q}_{p}\right)$ is skewsymmetric.

Property 3: The Coriolis and centripetal matrix $\left|C_{p}\left(q_{p}, \dot{q}_{p}\right)\right| \leq \kappa\left|\dot{q}_{p}\right|$ for $\kappa>0$ and $\left|\dot{q}_{p}\right| \leq \dot{q}_{p_{\max }}$ for each joint.

Property 4: The system dynamics of (10) can be linearly parameterized by a model regressor matrix $Y\left(q_{p}, \dot{q}_{p}, \ddot{q}_{p}\right) \in$ $\Re^{n \times r}$ and a parameter vector $p \in \Re^{r \times 1}$ as

$$
M_{p}\left(q_{p}\right) \ddot{q}_{p}+C_{p}\left(q_{p}, \dot{q}_{p}\right) \dot{q}_{p}+g_{p}\left(q_{p}\right)=Y\left(q_{p}, \dot{q}_{p}, \ddot{q}_{p}\right) p
$$

where $r$ and $n$ are the number of parameter vector elements and number of the joints respectively. In this paper, the prosthetic ankle is passive while the human one is obviously actuated, resulting in a two-domain hybrid asymmetric human/prosthesis system. Fig. 2(b) shows this two-domain system with one domain for human stance and the other for prosthetic stance.

\section{Problem Statement}

The scope of this paper encompasses control of the prosthetic knee joint shown in Fig. 2(a) using the three proposed controllers introduced in Section III. These model-based controllers are presented as a means of addressing the limitations of the VI and PD controllers. Using the proposed controllers, we formally present stability and robustness of the closed-loop system in the presence of system uncertainties and disturbances. We also greatly improve prosthesis knee tracking performance compared to the RSC, RPC, VI, and PD controllers. We finally verify our controllers on AMPRO3 in an outdoor test with three subjects walking on different flat and uneven surfaces.

The proposed controllers use only $S_{c}=\left\{q_{c}, q_{c}^{d}\right\}$ and hip information from the human/prosthesis system without any information about the user and prosthesis dynamics. From $S_{c}$ and using a linear transformation in Table I, $S_{p}=\left\{q_{p}, q_{p}^{d}\right\}$ can be computed. In Table I, $q_{p}^{d}$ is the desired trajectory for $q_{p}$; $p_{\text {hip }_{x}}$ and $p_{\text {hip }}$ are horizontal and vertical positions of the hip; 
TABLE I

LINEAR TRANSFORMATION BETWEEN HIP INFORMATION AND COORDinATES OF THE HEALTHY Body IN FIG. 2(a), AND LOCAL Degrees of Freedom of the Prosthesis Model in Fig. 3

\begin{tabular}{l|l}
\hline \multicolumn{1}{c|}{ Stance phase } & \multicolumn{1}{c}{ Swing phase } \\
\hline \hline$q_{p 1}=p_{\text {hip }_{x}}$ & $q_{p 1}=p_{\text {hip }_{x}}$ \\
$q_{p 2}=p_{h i p_{z}}-L_{z}$ & $q_{p 2}=p_{h i p_{z}}-L_{z}$ \\
$q_{p 3}=-q_{s k}+\pi / 2$ & $q_{p 3}=-q_{s k}+q_{s h}-q_{n s h}+\pi / 2$ \\
$q_{p 4}=q_{s k}$ & $q_{p 4}=q_{n s k}$ \\
\hline
\end{tabular}

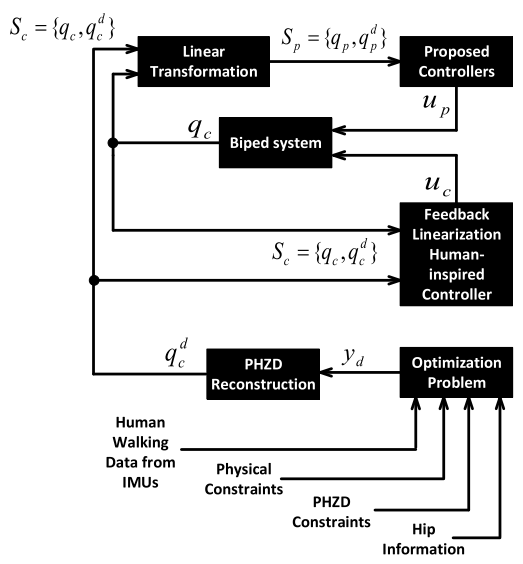

Fig. 4. Proposed structure for the biped system.

and $L_{z}$ denotes vertical distance between the world frames $O_{c} 0$ and $O_{0}$. The set $S_{p}$ is then used by the controllers to generate prosthetic knee torque $u_{p}$. To understand how the control signal remains correctly defined during stance and nonstance phases and how $u_{c}$ can be obtained from $u_{p}$, readers are referred to [20]. See Fig. 4 for the proposed control architecture.

\section{Proposed Prosthesis Controllers}

This section presents three different model-based controllers, ADC, RSAC, and AIC, to control the prosthetic knee joint while the other joints are controlled by a feedback linearization human-inspired controller [27]. In practice, all joints are controlled by the amputee other than the active prosthetic knee. This framework results in convergence of the outputs of the human/prosthesis system $y_{a}$ to the desired ones $y_{d}$ exponentially and provides stable and human-like walking.

\section{A. Adaptive Controller}

Robustness to only parametric uncertainties, no unmodeled dynamics, and zero disturbances.

Adaptive control implements learning and adaptation using online parameter vector $\left(p \in \mathfrak{R}^{r \times 1}\right)$ estimation in the control structure. The ADC aims to enhance tracking performance using estimation of the unknown prosthetic leg parameters while not considering unmodeled dynamics or disturbances $\left(d_{p}(t)=0\right)$. In this section, a direct ADC, which is a combination of a PD controller and tracking-error-based (TEB) adaptation mechanism, is presented [35].

Given Property 4, the system dynamics of (10) can be linearly parameterized as

$$
M_{p}\left(q_{p}\right) \ddot{q}_{p}+C_{p}\left(q_{p}, \dot{q}_{p}\right) \dot{q}_{p}+g_{p}\left(q_{p}\right)=Y\left(q_{p}, \dot{q}_{p}, \ddot{q}_{p}\right) p .
$$

Defining an error vector $s=\dot{e}+\lambda e$ and signal vector $v=$ $\dot{q}_{p}^{d}-\lambda e$, the ADC control law can be expressed as [36], [37]

$$
u_{p_{\mathrm{ADC}}}=Y\left(q_{p}, \dot{q}_{p}, v, \dot{v}\right) \hat{p}-K_{d_{\mathrm{ADC}}} s
$$

where $e=q_{p}-q_{p}^{d}$ and $\lambda=\operatorname{diag}\left(\lambda_{1}, \lambda_{2}, \ldots, \lambda_{n}\right), \lambda_{i}>0 ; K_{d_{\mathrm{ADC}}}$ is a diagonal matrix with positive elements; $Y\left(q_{p}, \dot{q}_{p}, v, \dot{v}\right)$ is the acceleration-free version of $Y\left(q_{p}, \dot{q}_{p}, \ddot{q}_{p}\right)$ [20]; and $\hat{p}$ is adjusted based on system joint measurements and the adaptation mechanism. Substituting (13) into (10) in the absence of nonparametric uncertainties and other disturbances, we have the closed-loop system

$$
M_{p}\left(q_{p}\right) \dot{s}+C_{p}\left(q_{p}, \dot{q}_{p}\right) s+K_{d_{\mathrm{ADC}}} s=Y\left(q_{p}, \dot{q}_{p}, v, \dot{v}\right) \tilde{p}
$$

where $\tilde{p}$ is the parameter vector estimation error. Consider the Lyapunov function candidate [36]

$$
V_{\mathrm{ADC}}(s, \tilde{p})=\frac{1}{2}\left(s^{T} M_{p}\left(q_{p}\right) s+\tilde{p}^{T} \gamma \tilde{p}\right)
$$

where $V_{\mathrm{ADC}}$ is a function of $s$ and $\tilde{p}$, and $\gamma$ is a design parameter such that $\gamma=\operatorname{diag}\left(\gamma_{1}, \gamma_{2}, \ldots, \gamma_{r}\right), \gamma_{i}>0$.

Theorem 1: Given the Lyapunov function $V_{\mathrm{ADC}}(s, \tilde{p})$ of (15), and the ADC control law of (13) in conjunction with the adaptation law $\dot{\hat{p}}_{1}=-\gamma^{-1} Y^{T}\left(q_{p}, \dot{q}_{p}, v, \dot{v}\right) s$, we obtain $\dot{V}_{\mathrm{ADC}}(s, \tilde{p}) \rightarrow 0$ as $t \rightarrow \infty$, which implies asymptotic stability of the closed-loop system, implying $s \rightarrow 0$ and $q_{p} \rightarrow q_{p}^{d}$ for all $\tilde{p} \in \mathfrak{R}^{r}$.

Barbalat's lemma [36] is used to prove Theorem 1.

Barbalat's Lemma: If a candidate Lyapunov function $V(t, x)$ satisfies the conditions: 1) $V(t, x)$ is lower-bounded; 2) $\dot{V}(t, x)$ is negative semi-definite; and 3) $\ddot{V}(t, x)$ is bounded, then $\dot{V}(t, x) \rightarrow 0$ as $t \rightarrow 0$.

Proof of Theorem 1: Taking the time derivative of (15), substituting the closed-loop system of (14), and using Property 2 $\left(s^{T}\left(\dot{M}_{p}\left(q_{p}\right)-2 C_{p}\left(q_{p}, \dot{q}_{p}\right)\right) s=0\right)$ yields

$$
\dot{V}_{\mathrm{ADC}}(s, \tilde{p})=-s^{T} K_{d_{\mathrm{ADC}}} s+s^{T} Y\left(q_{p}, \dot{q}_{p}, v, \dot{v}\right) \tilde{p}+\dot{\tilde{p}}^{T} \gamma \tilde{p} .
$$

The adaptation law $\dot{\hat{p}}_{1}=-\gamma Y^{T}\left(q_{p}, \dot{q}_{p}, v, \dot{v}\right) s$ yields

$$
\dot{V}_{\mathrm{ADC}}(s, \tilde{p})=-s^{T} K_{d_{\mathrm{ADC}}} s .
$$

Since $\dot{V}_{\mathrm{ADC}}(s, \tilde{p})$ of (17) is negative semi-definite, using Barbalat's lemma [36] it can be shown that $\dot{V}_{\mathrm{ADC}}(s, \tilde{p}) \rightarrow 0$. Since $V_{\mathrm{ADC}}(s, \tilde{p})$ of (15) is lower-bounded and (17) implies $V_{\mathrm{ADC}}(t) \leq V(0), V_{\mathrm{ADC}}(s, \tilde{p})$ and in turn $s$ and $\hat{p}$ are bounded. To check if $\dot{V}_{\mathrm{ADC}}(s, \tilde{p})$ is uniformly continuous in time, the second derivative of (17) is calculated as

$$
\ddot{V}_{\mathrm{ADC}}(s, \tilde{p})=-s^{T} K_{d_{\mathrm{ADC}}} \dot{s} .
$$

Substituting (14) into (18) yields

$$
\begin{aligned}
\ddot{V}_{\mathrm{ADC}}(s, \tilde{p})= & -2 s^{T} K_{d_{\mathrm{ADC}}} M_{p}^{-1}\left(q_{p}\right)\left(-C_{p}\left(q_{p}, \dot{q}_{p}\right) s-K_{\left.d_{\mathrm{ADC}} s\right)}\right. \\
& +M_{p}^{-1}\left(q_{p}\right) Y\left(q_{p}, \dot{q}_{p}, v, \dot{v}\right) \tilde{p} .
\end{aligned}
$$


As shown above, $s$ and $\hat{p}$ are bounded. Boundedness of $s$ implies that $e$ and $\dot{e}$ are bounded. Since all reference trajectories $q_{p}^{d}$ are bounded, $v, \dot{v}, q_{p}, \dot{q}_{p}$ and in turn $Y\left(q_{p}, \dot{q}_{p}, v, \dot{v}\right)$ are all bounded, so it can be concluded that $\ddot{V}_{\mathrm{ADC}}(s, \tilde{p})$ is bounded. Because all premises in Barbalat's lemma are satisfied, $\dot{V}_{\mathrm{ADC}}(s, \tilde{p}) \rightarrow 0$ as $t \rightarrow \infty$, which implies that the ADC guarantees asymptotic stability of the closed-loop system showing that $s \rightarrow 0$ and $q_{p} \rightarrow q_{p}^{d}$ for all $\tilde{p} \in \mathfrak{R}^{r}$.

\section{B. Robust Sliding Mode Adaptive Controller}

Robustness to all parametric uncertainties, neglected dynamics, and nonzero disturbances $d_{p}(t)$.

Since neglected dynamics and disturbances may degrade the performance of the closed-loop system, a robust control component should be added to the ADC to compensate for these effects. The learning nature of ADCs along with the existence of both disturbances and nonparametric uncertainties motivate the combination of the ADC in Section III-A and the RSC in [20] to build the RSAC.

Using the same sliding surface $s$, signal vector $v$, and controller regressor $Y\left(q_{p}, \dot{q}_{p}, v, \dot{v}\right)$, the control law is designed as in [37] to yield

$$
u_{p_{\mathrm{RSAC}}}=Y\left(q_{p}, \dot{q}_{p}, v, \dot{v}\right) \hat{p}-K_{d_{\mathrm{RSAC}}} \operatorname{sat}(s / \operatorname{diag}(\phi))
$$

where $\phi$ represents the saturation function such that $\phi=\operatorname{diag}\left(\phi_{1}, \phi_{2}, \ldots, \phi_{n}\right), \phi_{i}>0$; and $K_{d_{\mathrm{RSAC}}}$ is a diagonal matrix with positive elements. Note that the division and saturation operations for $s$ and $\operatorname{diag}(\phi)$ in the term $\operatorname{sat}(s / \operatorname{diag}(\phi))$ are interpreted element-wise and $\operatorname{diag}(\phi)$ is an $n$-element vector. $K_{d 2}$ is a diagonal matrix with positive diagonal elements and $\hat{p}$ is estimated by a TEB adaptation law. Substituting (20) into (10) yields

$$
\begin{aligned}
& M_{p}\left(q_{p}\right) \dot{s}+C_{p}\left(q_{p}, \dot{q}_{p}\right) s+K_{d_{\mathrm{RSAC}}} \operatorname{sat}(s / \operatorname{diag}(\phi)) \\
& \quad=Y\left(q_{p}, \dot{q}_{p}, v, \dot{v}\right) \tilde{p} .
\end{aligned}
$$

To prevent unfavorable parameter drift in the RSAC, the following nonscalar boundary layer trajectory $s_{\Delta}$ is suggested [37]

$$
s_{\Delta}= \begin{cases}0 & \text { if }|s| \leq \operatorname{diag}(\phi) \\ s-\phi \operatorname{sat}(s / \operatorname{diag}(\phi)) & \text { if }|s|>\operatorname{diag}(\phi)\end{cases}
$$

where $s_{\Delta}$ is an $n$-element vector; and $\phi$ is the boundary layer thickness. The reason for incorporating $s_{\Delta}$ in the control structure is three-fold: 1) to stop the TEB adaptation mechanism in the boundary layer $(|s| \leq \operatorname{diag}(\phi)) ; 2)$ to tradeoff control chattering and tracking performance; and 3) to bound all error trajectories in the boundary layers. This way, the RSAC shows robustness to parametric and nonparametric uncertainties in addition to disturbances while the error trajectories of the system converge to the boundary layer.

To prove the stability of the closed-loop system in the presence of both parametric and nonparametric uncertainties, $Y\left(q_{p}, \dot{q}_{p}, v, \dot{v}\right)$ and $\tilde{p}$ are split into two parts as

$$
Y=\left[\begin{array}{ll}
Y_{2} & Y_{\mathrm{ud}}
\end{array}\right], \quad \tilde{p}=\left[\begin{array}{ll}
\tilde{p}_{2}^{T} & \tilde{p}_{\mathrm{ud}}^{T}
\end{array}\right]
$$

where $Y_{2}$ is the modeled regressor and $\tilde{p}_{2}$ is its parameter vector, and $Y_{\text {ud }}$ and $\tilde{p}_{\text {ud }}$ are associated with unmodeled dynamics of the prosthetic leg. Consider the following Lyapunov function as a function of $s_{\Delta}$ and $\tilde{p}$ (as inspired from [37]):

$$
V_{\mathrm{RSAC}}\left(s_{\Delta}, \tilde{p}_{2}\right)=\frac{1}{2}\left(s_{\Delta}^{T} M_{p}\left(q_{p}\right) s_{\Delta}\right)+\frac{1}{2}\left(\tilde{p}_{2}^{T} \gamma \tilde{p}_{2}\right)
$$

Theorem 2: Assume that $\left|\left(Y_{\mathrm{ud}} \tilde{p}_{\mathrm{ud}}\right)_{i}\right| \leq P_{i},\left|d_{p_{i}}(t)\right| \leq D_{i}$, and $P_{i}, D_{i}>0$ for $i=1, \ldots, n$. Define $F_{m}=\max \left(P_{i}+D_{i}\right)$. Given the Lyapunov function $V_{\mathrm{RSAC}}\left(s_{\Delta}, \tilde{p}_{2}\right)$ of (24), the RSAC control law of (20), and the adaptation mechanism $\dot{\hat{p}}_{2}=-\gamma^{-1} Y_{2}^{T}\left(q_{p}, \dot{q}_{p}, v, \dot{v}\right) s_{\Delta}$, if control gain $K_{d_{\mathrm{RSACi}}} \geq F_{m}+\Lambda_{m}-\kappa \dot{q}_{p_{\max }} \phi_{i}$ with $\kappa, \Lambda_{m}>0$, then $\dot{V}_{\mathrm{RSAC}}\left(s_{\Delta}, \tilde{p}_{2}\right) \rightarrow 0$ as $t \rightarrow \infty$ for all $p \in \mathfrak{R}^{r}$ and $s(0) \in \mathfrak{R}^{n}$, which implies that $s_{\Delta} \rightarrow 0,\left|s_{i}\right| \leq \phi_{i}$, and $e \leq \phi_{i} / \lambda_{i}$.

Proof of Theorem 2: Noting that $\dot{s}_{\triangle}=\dot{s}$ if outside the boundary layer, and substituting the error dynamics of (21) into the derivative of (24) gives

$$
\begin{aligned}
\dot{V}_{\mathrm{RSAC}}\left(s_{\Delta}, \tilde{p}_{2}\right)= & -s_{\Delta}^{T} C_{p}\left(q_{p}, \dot{q}_{p}\right) s+\frac{1}{2}\left(s_{\Delta}^{T} \dot{M}_{p}\left(q_{p}\right) s_{\Delta}\right) \\
& -s_{\Delta}^{T} K_{d_{\mathrm{RSAC}}} \operatorname{sat}(s / \operatorname{diag}(\phi))+s_{\Delta}^{T} Y_{\mathrm{ud}} \tilde{p}_{\mathrm{ud}} \\
& +\left(\dot{\tilde{p}}_{2}^{T} \gamma+s_{\Delta}^{T} Y_{2}\right) \tilde{p}_{2}+s_{\Delta}^{T} d_{p}(t) .
\end{aligned}
$$

Choosing the TEB adaptation law as $\dot{\hat{p}}_{2}=-\gamma^{-1} Y_{2}^{T} s_{\Delta}$, substituting $s=s_{\Delta}+\phi \operatorname{sat}(s / \operatorname{diag}(\phi))$ from (22) into (25) if outside the boundary layer, and applying Property 2 $\left(s_{\Delta}^{T}\left(\dot{M}_{p}\left(q_{p}\right)-2 C_{p}\left(q_{p}, \dot{q}_{p}\right)\right) s_{\Delta}=0\right)$ yields [38], [39]

$$
\begin{aligned}
\dot{V}_{\mathrm{RSAC}}\left(s_{\Delta}, \tilde{p}_{2}\right)= & -s_{\Delta}^{T}\left(C_{p}\left(q_{p}, \dot{q}_{p}\right) \phi+K_{d_{\mathrm{RSAC}}}\right) \operatorname{sat}(s / \operatorname{diag}(\phi)) \\
& +s_{\Delta}^{T}\left(Y_{\mathrm{ud}} \tilde{p}_{\mathrm{ud}}+d_{p}(t)\right) .
\end{aligned}
$$

Tuning $K_{d_{\mathrm{RSAC}}}$ and $\phi$ so $C_{p}\left(q_{p}, \dot{q}_{p}\right) \phi+K_{d_{\mathrm{RSAC}}} \geq K_{m} I$ with $K_{m}>0$, and noting that $s_{\Delta}^{T} \operatorname{sat}(s / \operatorname{diag}(\phi))=\left\|s_{\Delta}\right\|_{1}$ gives

$$
\dot{V}_{\mathrm{RSAC}}\left(s_{\Delta}, \tilde{p}_{2}\right) \leq-K_{m}\left\|s_{\Delta}\right\|_{1}+s_{\Delta}^{T}\left(Y_{\mathrm{ud}} \tilde{p}_{\mathrm{ud}}+d_{p}(t)\right) .
$$

Condition $C_{p} \phi+K_{d_{\mathrm{RSAC}}} \geq K_{m} I$ can be restated as $K_{d_{\mathrm{RSACi}}} \geq F_{m}+\Lambda_{m}-\kappa \dot{q}_{p_{\max }} \phi_{i}$ (Property 3) for each joint with $\kappa$ as a positive scalar and $\dot{q}_{p_{\max }}$ as the maximum prosthesis joint velocity. Assume $\left|\left(Y_{\mathrm{ud}} \tilde{p}_{\mathrm{ud}}+d_{p}(t)\right)_{i}\right| \leq P_{i}+D_{i}$ for each joint. Then, defining $F_{m}=\max \left(P_{i}+D_{i}\right), K_{m}=F_{m}+\Lambda_{m}$ with $\Lambda_{m}>0$, and noting that $s_{\Delta}^{T} F_{m}$ is upper bounded by $F_{m}\left\|s_{\Delta}\right\|_{1}$ yields

$$
\dot{V}_{\mathrm{RSAC}}\left(s_{\Delta}, \tilde{p}_{2}\right) \leq-\Lambda_{m}\left\|s_{\Delta}\right\|_{1} .
$$

As (28) is negative semi-definite outside the boundary layer, using Barbalat's lemma, asymptotic convergence of $\dot{V}_{\mathrm{RSAC}}\left(s_{\Delta}, \tilde{p}_{2}\right)$ to zero and boundedness of the error trajectories can be guaranteed. Premises I and II of Barbalat's lemma can be verified from (24) and (28), and in turn $\dot{V}_{\mathrm{RSAC}}\left(s_{\Delta}, \tilde{p}_{2}\right)$ and all terms in it, namely, $\tilde{p}_{2}$ and $s_{\Delta}$, are bounded. As $p_{2}$ is constant, boundedness of $\tilde{p}_{2}$ shows that $\hat{p}_{2}$ is bounded. Also, since $s_{\Delta}$ is bounded, $s$ is bounded. Taking the second derivative of (28) yields

$$
\begin{aligned}
\ddot{V}_{\mathrm{RSAC}}\left(s_{\Delta}, \tilde{p}_{2}\right) & \leq-\Lambda_{m} \frac{d}{d t}\left\|s_{\Delta}\right\|_{1}= \pm \Lambda_{m} \sum \frac{s_{\Delta} \dot{s}_{\Delta}}{\left|s_{\Delta}\right|} \\
& = \pm \Lambda_{m} \sum \dot{s} .
\end{aligned}
$$


Substituting $\dot{s}$ from (21) into (29) and noting that $s_{\Delta} \neq 0$ outside the boundary layer gives

$$
\begin{aligned}
& \ddot{V}_{\mathrm{RSAC}}\left(s_{\Delta}, \tilde{p}_{2}\right) \\
& \leq \pm \Lambda_{m} \sum M_{p}^{-1}\left(q_{p}\right)\left\{-K_{d_{\mathrm{RSAC}}} \operatorname{sat}(s / \operatorname{diag}(\phi))+Y_{\mathrm{ud}} \tilde{p}_{\mathrm{ud}}\right. \\
& \left.+Y_{2} \tilde{p}_{2}+d_{p}(t)-C_{p}\left(q_{p}, \dot{q}_{p}\right) s\right\}
\end{aligned}
$$

As shown above, since $s$ and $\tilde{p}_{2}$ are bounded, and $\left|\left(Y_{\mathrm{ud}} \tilde{p}_{\mathrm{ud}}+d_{p}(t)\right)_{i}\right| \leq P_{i}+D_{i}$, boundedness of $\ddot{V}_{\mathrm{RSAC}}\left(s_{\Delta}, \tilde{p}_{2}\right)$ is concluded. Therefore, as premises I-III in Barbalat's lemma are satisfied, $\dot{V}_{\mathrm{RSAC}}\left(s_{\Delta}, \tilde{p}_{2}\right) \rightarrow 0$ as $t \rightarrow 0$ and in turn $s_{\Delta} \rightarrow 0$, which means all error trajectories are attracted by the boundary layer if starting outside the boundary layer. On the other hand, inside the boundary layer, $s_{\Delta}=0$ and $s$ remains in the layer.

Remark 1: It should be noted that convergence of the error trajectories to the boundary layer does not imply asymptotic convergence of $\tilde{p}_{2}$. Estimated parameter vector $\hat{p}_{2}$ asymptotically converges to its true value if $Y\left(q_{p}, \dot{q}_{p}, v, \dot{v}\right)$ is persistently exciting and uniformly continuous [35].

\section{Adaptive Integral Controller}

Robustness to only parametric uncertainties, no unmodeled dynamics, and zero disturbances.

To enhance tracking performance of the ADC from Section III-A, a different time-varying sliding surface is proposed which is the weighted sum of the position error, the velocity error, and the integral of the position error [36]

$$
s_{I}=\dot{e}+2 \lambda e+\lambda^{2} \int_{0}^{t} e d t .
$$

To obtain the same control law structure and error dynamics of the ADC as presented in (13) and (14) respectively, signal vector $v_{I}$ is defined as

$$
v_{I}=\dot{q}_{p}^{d}-2 \lambda e-\lambda^{2} \int_{0}^{t} e d t .
$$

Using these error and signal vectors, the AIC control law and its error dynamics can be written as

$$
\begin{aligned}
u_{p_{\mathrm{AIC}}}= & Y\left(q_{p}, \dot{q}_{p}, v_{I}, \dot{v}_{I}\right) \hat{p}-K_{d_{\mathrm{AIC}}} s_{I} M_{p}\left(q_{p}\right) \dot{s}_{I} \\
& +C_{p}\left(q_{p}, \dot{q}_{p}\right) s_{I}+K_{d_{\mathrm{AIC}}} s_{I} \\
= & Y\left(q_{p}, \dot{q}_{p}, v_{I}, \dot{v}_{I}\right) \tilde{p}
\end{aligned}
$$

where $K_{d_{\mathrm{AIC}}}$ is a diagonal matrix with positive elements. Consider the following Lyapunov function, which is a function of $s_{I}$ and $\tilde{p}$ :

$$
V_{\mathrm{AIC}}\left(s_{I}, \tilde{p}\right)=\frac{1}{2}\left(s_{I}^{T} M_{p}\left(q_{p}\right) s_{I}+\tilde{p}^{T} \gamma \tilde{p}\right) .
$$

Theorem 3: Given the Lyapunov function $V_{\mathrm{AIC}}\left(s_{I}, \tilde{p}\right)$ of (34), and the AIC control law of (33) in conjunction with the adaptation law $\dot{\hat{p}}_{3}=-\gamma^{-1} Y^{T}\left(q_{p}, \dot{q}_{p}, v_{I}, \dot{v}_{I}\right) s_{I}$, we obtain $\dot{V}_{\text {AIC }}\left(s_{I}, \tilde{p}\right) \rightarrow 0$ as $t \rightarrow \infty$, which implies asymptotic stability of the closed-loop system, showing $s_{I} \rightarrow 0$ and $e \rightarrow 0$ for all $\tilde{p} \in \mathfrak{R}^{r}$.
Proof of Theorem 3: Similar to the proof of Theorem 1, taking the derivative of (34), substituting the error dynamics of (33) into the result, applying the adaptation mechanism $\dot{\hat{p}}_{3}=-\gamma^{-1} Y^{T}\left(q_{p}, \dot{q}_{p}, v_{I}, \dot{v}_{I}\right) s_{I}$, and using Property 2 yields

$$
\dot{V}_{\mathrm{AIC}}\left(s_{I}, \tilde{p}\right)=-s_{I}^{T} K_{d_{\mathrm{AIC}}} s_{I} .
$$

Because $\dot{V}_{\mathrm{AIC}}\left(s_{I}, \tilde{p}\right)$ is negative semi-definite, $V_{\mathrm{AIC}}$ is lowerbounded, and $\ddot{V}_{\text {AIC }}$ is bounded, using Barbalat's lemma as in Section III-A demonstrates that $\dot{V}_{\mathrm{AIC}}\left(s_{I}, \tilde{p}\right) \rightarrow 0$ as $t \rightarrow \infty$. This implies that the AIC guarantees asymptotic stability of the closed-loop system, showing $s_{I} \rightarrow 0$ and $q_{p} \rightarrow q_{p}^{d}$ for all $\tilde{p} \in \mathfrak{R}^{r}$.

Remark 2: The regressor matrix $Y\left(q_{p}, \dot{q}_{p}, v, \dot{v}\right)$ is joint acceleration-independent resulting in an acceleration-free control law and adaptation mechanism for all controllers. All the proposed controllers only need joint position and velocity $\left(q_{p}, \dot{q}_{p}\right)$, and desired joint position, velocity, and acceleration $\left(q_{p}^{d}, \dot{q}_{p}^{d}, \ddot{q}_{p}^{d}\right)$. This way, there is no need to measure joint acceleration and no concern about measurement noise.

\section{Overview of $R S C$ and $R P C$}

The framework of RSC and RPC [20] are summarized as follows.

1) Robust Sliding Mode Controller: The RSC provides robustness to all parametric uncertainties, neglected dynamics, and existing disturbances $d_{p}(t)$. The RSC is obtained as [20], [36]

$$
u_{p_{\mathrm{RSC}}}=Y\left(q_{p}, \dot{q}_{p}, v, \dot{v}\right) \hat{p}-K_{d_{\mathrm{RSC}}} \operatorname{sat}(s / \operatorname{diag}(\phi))
$$

where $K_{d_{\mathrm{RSC}}}$ is a diagonal matrix and $\hat{p}$ is our best knowledge of the parameter vector. Consider the following Lyapunov function:

$$
V_{\mathrm{RSC}}\left(s_{\Delta}\right)=\frac{1}{2}\left(s_{\Delta}^{T} M_{p}\left(q_{p}\right) s_{\Delta}\right) .
$$

This yields the following result, wherein a detailed proof can be found in [20].

Theorem 4: Assume that $\left|\left(Y\left(q_{p}, \dot{q}_{p}, v, \dot{v}\right) \tilde{p}\right)_{i}\right| \leq Q_{i}$ and $\left|d_{p_{i}}(t)\right| \leq D_{i}$ for $i=1, \ldots, n$ and $Q_{i}, D_{i}>0$. Define $F_{m}=\max \left(Q_{i}+D_{i}\right)$. Given the Lyapunov function $V_{\mathrm{RSC}}\left(s_{\Delta}\right)$ of (37) and the RSC control law of (36), if $K_{d_{\mathrm{RSCi}}} \geq F_{m}+\Lambda_{m}-$ $\kappa \dot{q}_{p_{\max }} \phi_{i}$ with $\kappa, \Lambda_{m}>0$, then $\dot{V}_{\mathrm{RSC}}\left(s_{\Delta}\right) \rightarrow 0$ and $s_{\Delta} \rightarrow 0$ as $t \rightarrow \infty$ for all $p \in \Re^{r}$ and $s(0) \in \Re^{n}$, which implies $\left|s_{i}\right| \leq \phi_{i}$ and the error term $e \leq \phi_{i} / \lambda_{i}$.

2) Robust Passivity Controller: The RPC provides robustness to only parametric uncertainties and unmodeled dynamics [assuming $d_{p}(t)=0$ ]. The RPC is presented based on passivity in parameters and a switching control law

$$
u_{p_{\mathrm{RPC}}}=Y\left(q_{p}, \dot{q}_{p}, v, \dot{v}\right) \hat{p}-K_{d_{\mathrm{RPC}}} s
$$

where $K_{d_{\mathrm{RPC}}}$ is a diagonal matrix with positive diagonal entries and $\hat{p}$ is derived based on a switching control law [20], [28].

Consider the following Lyapunov function:

$$
V_{\mathrm{RPC}}(s, e)=\frac{1}{2}\left(s^{T} M_{p}\left(q_{p}\right) s+e^{T} \lambda K_{d_{\mathrm{RPC}}} e\right) .
$$

This yields the following result, wherein a detailed proof can be found in [20]. 

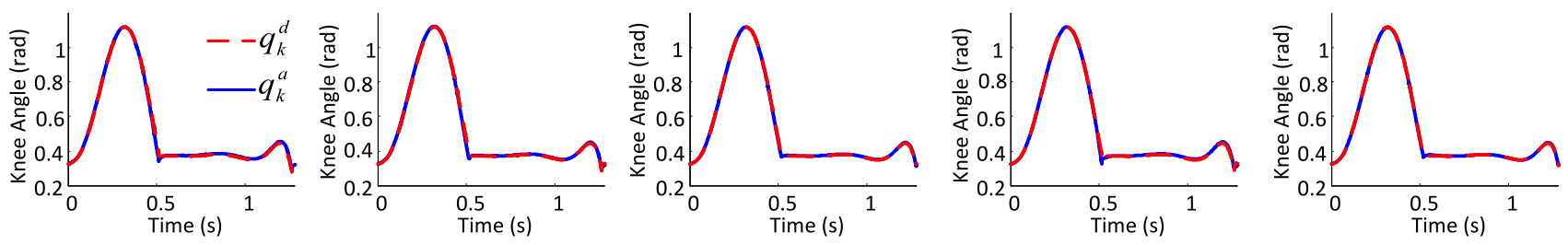

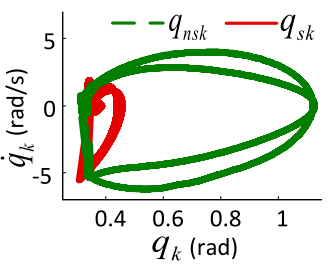

RSC

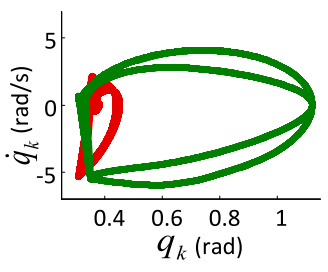

RPC

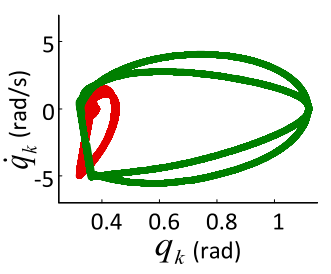

ADC

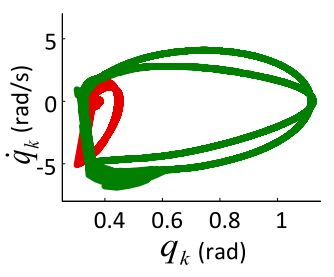

RSAC

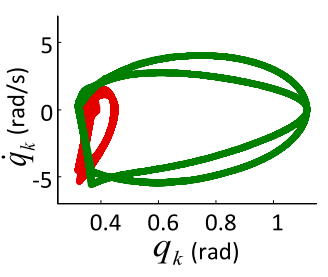

AIC

Fig. 5. Tracking performance of the prosthetic knee joint over two steps for different model-based controllers along with their phase portraits over 40 steps.

Theorem 5: Under certain assumptions and definitions, and given the Lyapunov function $V_{\mathrm{RPC}}(s, e)$ of (39), the RPC control law of (38), $\dot{V}_{\mathrm{RPC}}(s, e) \rightarrow 0$ as $t \rightarrow \infty$, which implies boundedness of all tracking error trajectories.

\section{Simulation Results on Virtual HUMAN/ROBOT SYSTEM}

In this section, we evaluate all three proposed controllers for the walking biped platform shown in Fig. 2(a), while both body and prosthesis device parameters are unknown to the controller. As mentioned above, the proposed controllers are only used to control the prosthetic knee while the rest of the joints are controlled by the feedback linearization human-inspired controller [27]. This coincides with the fact that in practice, all joints can be controlled by the human except amputated joints. Recall that in Section II, the desired prosthesis trajectory, $q_{p}^{d}$, was derived via optimization and the PHZD reconstruction procedure. All the proposed controllers are then compared to the RSC and RPC with regard to tracking performance and robustness to unexpected push and obstacle disturbances.

Remark 3: The design parameters of all model-based controllers are listed in Table II. We tune the design parameters of each controller to achieve the best tradeoff between tracking accuracy and robustness to disturbances $d_{p}(t)$ and neglected dynamics. Specifically, tuning the parameters for each controller is based on the following concepts: increasing $\lambda$ improves tracking; increasing $K_{d}$ enhances stability and robustness; decreasing $\gamma$ improves adaptation convergence rate; and $\phi$ and $\epsilon$ make a tradeoff between tracking and chattering.

\section{A. Tracking Performance and Human-Like Walking}

Fig. 5 illustrates prosthetic knee tracking performance for all proposed controllers as well as RSC and RPC over two steps. However, note that the entire simulation runs for 40 steps. It can be observed that all controllers track the desired trajectories in both stance and nonstance phase. Fig. 5 also provides phase portraits for the stance and nonstance knee joints over 40 steps. The phase portraits show convergence of
TABLE II

Design Parameters of the Model-Based Controllers in the SIMULATION AND EXPERIMENTS: (SIMULATION, EXPERIMENT)

\begin{tabular}{cccc}
\hline Controller & Parameter & Stance & Non-stance \\
\hline \hline \multirow{3}{*}{ ADC } & $\lambda$ & $(40 \mathrm{I}, 40 \mathrm{I})$ & $(40 \mathrm{I}, 35 \mathrm{I})$ \\
& $K_{d 1}$ & $(40 \mathrm{I}, 50 \mathrm{I})$ & $(40 \mathrm{I}, 35 \mathrm{I})$ \\
& $\gamma$ & $(0.01 \mathrm{I}, 0.01 \mathrm{I})$ & $(1000 \mathrm{I}, 1000 \mathrm{I})$ \\
\hline & $\lambda$ & $(40 \mathrm{I}, 50 \mathrm{I})$ & $(40 \mathrm{I}, 50 \mathrm{I})$ \\
RSAC & $K_{d 2}$ & $(100 \mathrm{I}, 100 \mathrm{I})$ & $(100 \mathrm{I}, 100 \mathrm{I})$ \\
& $\gamma$ & $(0.1 \mathrm{I}, 0.1 \mathrm{I})$ & $(1000 \mathrm{I}, 1000 \mathrm{I})$ \\
& $\phi$ & $(2 \mathrm{I}, 4 \mathrm{I})$ & $(2 \mathrm{I}, 4 \mathrm{I})$ \\
\hline \multirow{3}{*}{$\mathrm{AIC}$} & $\lambda$ & $(30 \mathrm{I}, 25 \mathrm{I})$ & $(30 \mathrm{I}, 35 \mathrm{I})$ \\
& $K_{d 3}$ & $(40 \mathrm{I}, 40 \mathrm{I})$ & $(40 \mathrm{I}, 40 \mathrm{I})$ \\
& $\gamma$ & $(0.1 \mathrm{I}, 0.01 \mathrm{I})$ & $(1000 \mathrm{I}, 1000 \mathrm{I})$ \\
\hline \multirow{3}{*}{$\mathrm{RSC}$} & $\lambda$ & $(50 \mathrm{I}, 40 \mathrm{I})$ & $(30 \mathrm{I}, 30 \mathrm{I})$ \\
& $K_{d 4}$ & $(50 \mathrm{I}, 40 \mathrm{I})$ & $(30 \mathrm{I}, 30 \mathrm{I})$ \\
& $\phi$ & $(0.5 \mathrm{I}, 1 \mathrm{I})$ & $(0.5 \mathrm{I}, 1 \mathrm{I})$ \\
\multirow{2}{*}{$\mathrm{RPC}$} & $\lambda$ & $(30 \mathrm{I}, 25 \mathrm{I})$ & $(10 \mathrm{I}, 10 \mathrm{I})$ \\
& $K_{d 5}$ & $(40 \mathrm{I}, 35 \mathrm{I})$ & $(20 \mathrm{I}, 20 \mathrm{I})$ \\
& $\epsilon$ & $(10000,10000)$ & $(10000,10000)$ \\
\hline
\end{tabular}

the controllers to a stable limit periodic orbit. It can also be seen from a careful inspection that the most consistent portraits belong to the ADC and AIC, showing the best tracking performance with more appropriate velocity at impact compared to other controllers. The impact upon foot strike induces a larger knee velocity change with the RSC and RPC (roughly $0.6 \mathrm{rad} / \mathrm{s}$ velocity jump when the ska is $0.35 \mathrm{rad}$ ) than the others. Furthermore, when impact occurs, the RSAC produces a higher negative knee velocity (approximately $1 \mathrm{rad} / \mathrm{s}$ higher velocity than the others at the end of swing phase), resulting in a highly variable phase portrait.

\section{B. Robustness Tests}

To test the stability of the virtual human/prosthesis system and quantify human/prosthesis system robustness in the presence of disturbances [unexpected pushes and obstacles as shown in Fig. 8(a)], two robustness tests are performed for all proposed controllers and the results are compared with the RSC and RPC. For the first robustness test, pushes are applied to the prosthetic leg in both $x$-direction and $z$-direction [with respect to the combined system world frame $O_{\mathrm{co}}=\left\{x_{\mathrm{co}}, y_{\mathrm{co}}, z_{\mathrm{co}}\right\}$ shown in Fig. 8(a) $]$. 

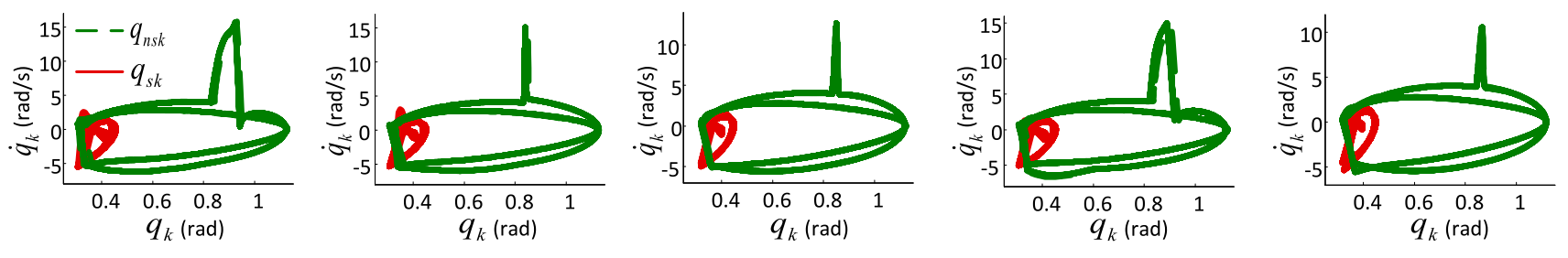

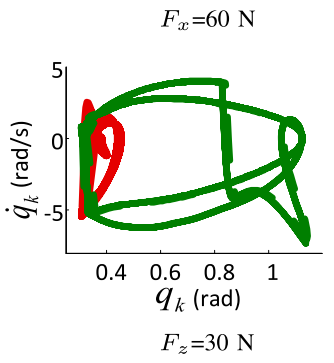

RSC

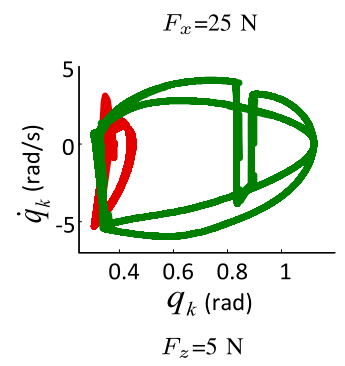

RPC

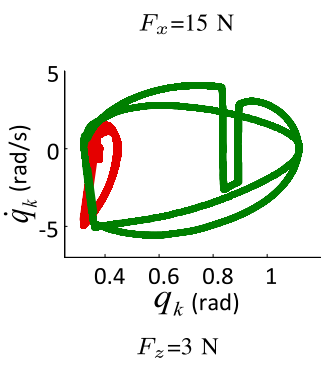

$\mathrm{ADC}$

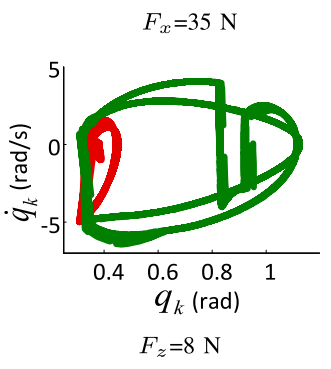

RSAC

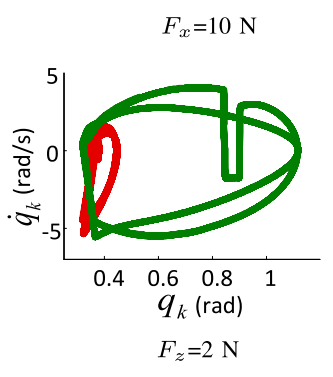

AIC

Fig. 6. Phase portrait of the knee joint over 40 steps with the applied impulse force at different levels, where $F_{x}$ and $F_{z}$ are $x$-direction and $z$-direction forces, respectively.

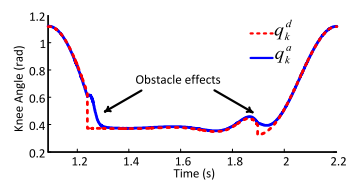

RSC, $20 \mathrm{~mm}$

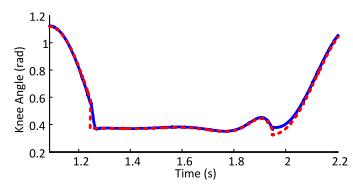

RPC, $15 \mathrm{~mm}$

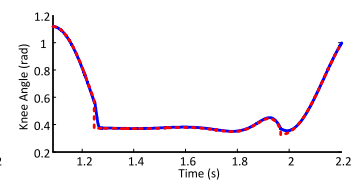

ADC, $15 \mathrm{~mm}$

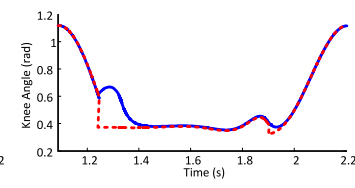

RSAC, $20 \mathrm{~mm}$

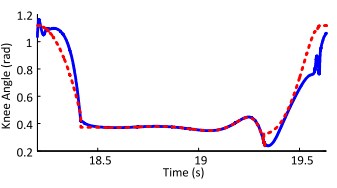

AIC, $5 \mathrm{~mm}$

Fig. 7. Prosthetic knee angle using different model-based controllers when the system walks over the obstacle.

1) Robustness to Impulse Force: For the first test, an $x$-direction impulse (lasting for $0.01 \mathrm{~s}$ ) is applied to the prosthetic leg for 40 steps each time it begins to swing forward. Fig. 6 depicts the phase portrait of the system under all control approaches during the test. Beginning with the RSC, the maximum tolerated force was determined to be $60 \mathrm{~N}$. It can be observed that the nonstance knee phase portrait deviates when the $x$-direction force is applied, but then smoothly converges to the cycle. This implies that the RSC can consistently tolerate the applied $x$-direction disturbance.

A similar test is also performed for the other controllers, but these controllers are not able to tolerate the same $60 \mathrm{~N}$ force in the $x$-direction, causing the system to fall at some point during the walking. The force of the impulse is then iteratively decreased with a resolution of $1 \mathrm{~N}$ until it can be tolerated. Fig. 6 shows convergence of all other controllers' phase portraits after reducing the force during each prosthetic swing for each individual controller.

Similarly, a $30 \mathrm{~N} z$-direction force is applied during prosthetic swing for all controllers for 40 steps. The RSC shows a good robustness to this force by completing 40 steps, while simulation with other controllers fails again. Fig. 6 demonstrates convergence of all controllers to the cycle when the $z$-direction force applied to the other controllers is reduced. Fig. 6 also shows a sharper velocity change on the phase portraits of the RPC, ADC, and AIC compared to the RSC and RSAC when $z$ - and $x$-direction forces are applied to the prosthetic leg.
2) Robustness to Obstacles: For the second robustness test, the human/prosthesis system is forced to walk over an obstacle for 40 steps. Fig. 7 demonstrates two steps of the actual and desired prosthesis knee angles captured during walking for all controllers at the point when the obstacle appears. For each controller, an unexpected obstacle appears each step during the prosthesis stance phase (see video available at [25]). From this figure, it is seen that the RSC and RSAC can overcome the $20 \mathrm{~mm}$ obstacle, while other controllers cannot walk over the same $20 \mathrm{~mm}$ obstacle. The height of the obstacle is iteratively decreased with a resolution of $1 \mathrm{~mm}$ until it can be walked over. It should be noted that when the biped walks over the obstacle, its next swing step tends toward greater knee extension no matter which controller is used.

To better characterize the controller responses, Fig. 7 shows arrows demonstrating the exact moment the obstacle is encountered. From this figure, it is easily observed that $\mathrm{ADC}$ and RPC quickly converge to the desired trajectory after encountering the obstacle, RSC and RSAC have a more sluggish response, and the AIC has the worst convergence.

3) Numerical Discussion: Table III lists root mean square error (RMSE) values for prosthetic knee tracking using all controllers and corresponding maximum disturbance tolerated in the robustness tests for 40 steps.

This table shows that the RSC provides the poorest knee tracking and the AIC provides the best. The AIC achieves $70 \%$ better tracking than the RSC. As tracking and robustness performances are conflicting objectives, the RSC outperforms 
TABLE III

Tracking Results and Robustness Performance For the Proposed Controllers as Well as the RSC AND RPC. The Best VALUE FOR EACH METRIC IS UNDERLINED

\begin{tabular}{cccccc}
\hline & RSC & RPC & ADC & RSAC & AIC \\
\hline \hline RMSE $_{40 \text { Steps }^{(}(\mathrm{rad})}$ & 0.0124 & 0.0111 & 0.0043 & 0.0059 & $\underline{0.0037}$ \\
MDT $_{\text {Force }}(\mathrm{N})$ & $\underline{60}$ & 25 & 15 & 35 & 10 \\
MDT $_{\text {Force }_{\mathbf{Z}}}(\mathrm{N})$ & $\underline{\underline{30}}$ & 5 & 3 & 8 & 2 \\
MDT $_{\text {Obstacle }}(\mathrm{mm})$ & $\underline{\underline{20}}$ & 15 & 15 & $\underline{20}$ & 5 \\
\hline
\end{tabular}

the other controllers with regard to robustness to pushes and obstacle disturbances, whereas the AIC ranks last in robustness.

According to Table III, the RSAC has the same level of robustness to obstacles as the RSC while performing second best in terms of robustness to pushes. The greater robustness of the RSC and RSAC in simulation is supported by the proofs in Section III. Recall that stability of RSC and RSAC is mathematically proven in the presence of time-varying disturbance $d_{p}(t)$, whereas stability of other proposed controllers cannot be guaranteed in the presence of external disturbances.

As also seen from the proofs in Section III, the AIC and ADC can only deal with parametric uncertainties and the RPC can only handle parametric uncertainties and unmodeled dynamics but not exogenous disturbances. However, the ADC, AIC, and RPC are still able to show some robustness to the disturbances. It should be noted that the AIC has the worst robustness to disturbances, whereas the best tracking of this controller comes from its integral operation.

According to Table III, the RSAC is able to achieve the best tradeoff between tracking and robustness, in line with its main goal of achieving acceptable tracking performance while providing good robustness to all parametric uncertainties, unmodeled dynamics, and disturbances. As seen from the tracking results and robustness tests, the RSC provides the best robustness and stability in the presence of disturbances, the AIC provides the best tracking performance, and the RSAC provides the best compromise between these goals.

\section{EXPERIMENTAL RESULTS ON AMPRO3}

In this section, all proposed controllers are tested experimentally using the powered self-contained transfemoral prosthesis AMPRO3 on a treadmill, as shown in Fig. 8(b), and compared with RSC, RPC, and two popular model-free controllers: VI and PD. Finally, outdoor tests are carried out to demonstrate walking in the real world.

\section{A. Detailed Description of AMPRO3 Mechanical Design}

This device has two $206 \mathrm{~W}$ brushless de motors (MOOG BN23) to actuate ankle and knee flexion/extension joints. A pair of torsion springs is incorporated between the harmonic gearbox and joints, resulting in series elastic actuators. The reason for using the torsion springs is three-fold: 1) to increase comfort for the user; 2) to prevent impacts from transferring directly to the gearboxes/motors, preventing damage; and 3) to improve energy efficiency and make walking speed acceleration easier.

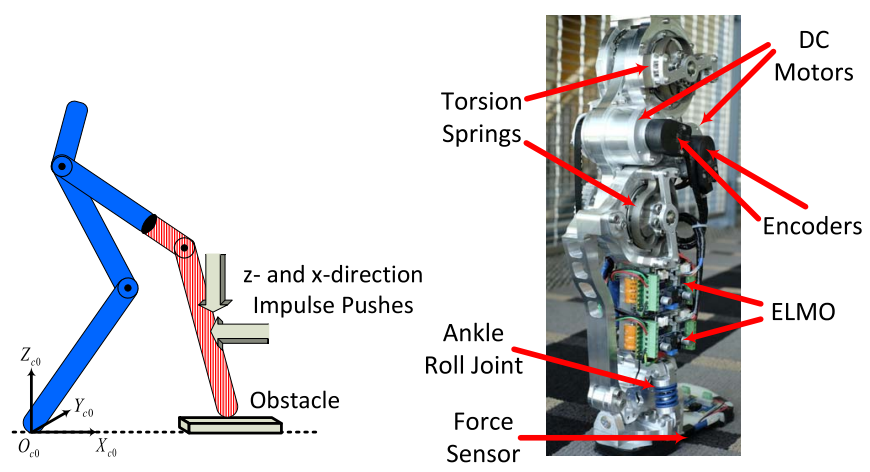

(a)

(b)

Fig. 8. (a) Robustness test with push and obstacle disturbances. (b) AMPRO3 device with its components.

In AMPRO3, motor and gearbox are separated from each other with a pulley-belt driven transmission system. The reason for this design is two-fold: 1) a greater gear reduction ratio can be achieved by choosing differently sized pulleys, resulting in a smaller and lighter harmonic gearbox and 2) a pulley-belt transmission isolates the motors from direct impact shocks. AMPRO3 uses two ELMO motion controllers (Gold Solo Whistle) to drive the motors with low-level torque control. Two encoders are used at both knee and ankle joints and connected to ELMO drives for motor control and joint feedback information.

A passive ankle roll joint is used for lateral ankle movement for comfort and for more natural three-dimensional gait. AMPRO3 uses a 9-cell Lipo battery (ThunderPower) to power the device. A 6-axis load cell is incorporated in series between the calf and the foot. Two flex force sensors are used for on and off ground contact conditions. The total weight of AMPRO3 is $5.95 \mathrm{Kg}$ (without knee adapter) and the total height is $451 \mathrm{~mm}$. AMPRO3 and its components are shown in Fig. 8(b) and further details about the device can be found in [32] and [40].

\section{B. Control Architecture}

The control architecture of the AMPRO3 device comprises three levels: 1) low-level control is handled by the ELMO motion drives to compensate for unmodeled dynamics such as friction, damping effects, and transmission dynamics of the motors; 2) mid-level control computes the input torques for the joints; and 3) high-level control is responsible for robot/human interaction. We code high-level controllers and trajectories in $\mathrm{C}++$ using robot operating system (ROS) packages. Trajectories and controller outputs are calculated by a single Beaglebone Black at $200 \mathrm{~Hz}$.

ROS includes many helpful modules for robotic control and data visualization (e.g., rviz). While ROS is widely used throughout multiagent robotic swarms and humanoid robots, in this paper, it is primarily used only for control loop parallelization and setting initial startup conditions. Upon startup, the ROS Master node populates the parameter server with initial conditions, facilitating quick debugging and allowing testing without a recompilation. There exist separate nodes for collecting sensor data, one each for the encoders and force sensors. 
The entire ROS implementation, including simple message passing between the sole control node and master node, operates natively on the BeagleBone single board computer. The most important portions of the control code are expressed as controller definitions in this paper, and other implementation details are derived from hardware libraries provided by the manufacturers.

\section{Test Procedure}

The functional reach test (FRT) and two-minute walk (2MW) test are two widely accepted tests for quantifying the performance of prostheses. The FRT is usually used to test for balance [41]. However, the primary investigation of this paper is not static posture stability, but rather prosthetic knee dynamics during walking. The $2 \mathrm{MW}$ test is a test of endurance which evaluates metabolic capacity [42]. Importantly, the main independent variable is often distance covered when walking. The intent of this paper is not to characterize the role of fatigue during walking, but rather to isolate the behaviors of the prosthesis controllers and their relationship to simulated values. Again, we elect not to perform the $2 \mathrm{MW}$ test because the objective is not to quantify the effects of fatigue on distance traveled. Instead, we choose to allow the subjects to walk for $2.5 \mathrm{~min}$ at a leisurely pace to allow them time to acclimate to the device. In this paper, the treadmill is speed-controlled for the purpose of test consistency. The representative periods of prosthesis data used in the comparisons are chosen for their consistency from step-to-step, as human model inputs in the simulation are also constant.

We test the proposed controllers ADC, RSAC, and AIC on AMPRO3 using an able-bodied subject on a treadmill and compare them with RSC, RPC, VI and PD controllers. It should be pointed out that in experiments, the prosthetic knee joint is controlled by the proposed controllers while the ankle joint is controlled by a PD controller to act like a passive joint [20]. During the treadmill experiments, an ablebodied subject wears the prosthesis with a bent-knee adapter as shown in Fig. 1 and walks on the treadmill for $2.5 \mathrm{~min}$ at $2 \mathrm{mph}$. For ease of implementation and reduction of noise, the hip horizontal, and vertical positions ( $q_{p 1}$ and $q_{p 2}$ respectively), and thigh angle $\left(q_{p 3}\right)$ of the prosthetic device modeled in Section II-B are considered to be their desired values in the controller rather than values from IMU measurements. Finally, three test subjects are asked to walk with AMPRO3 on level ground, uphill slopes, and downhill slopes in different outdoor environments as will be discussed at the end of this section.

\section{Implementation of the VI and PD Controllers}

1) Variable Impedance Controller: Because the VI controller is model-free, the prosthetic knee torque during a single stride (one gait cycle) can be generated using only local information of the knee joint modeled as series of passive impedance functions. In this approach, one stride is divided into several subphases, and each subphase is controlled by its own impedance controller. In this paper, one gait cycle is separated into three phases denoted $p \in\{1,2,3\}$, i.e., one for stance $\left(p^{1}\right)$, one for swing back $\left(p^{2}\right)$, and one for swing forward $\left(p^{3}\right)$. The prosthetic knee torque of the VI controller is generated from

$$
u_{V I}^{p}=k^{p}\left(q_{\mathrm{knee}}-q_{e}^{p}\right)+b^{p} \dot{q}_{\mathrm{knee}}
$$

where $q_{\text {knee }}$ and $\dot{q}_{\text {knee }}$ are angle and angular velocity of the prosthetic knee joint; $p$ is phase number; and $k^{p}, q_{e}^{p}$, and $b^{p}$ are phase-specific stiffness, damping, and equilibrium angle values respectively. Therefore, a total of nine parameters must be tuned for the VI controller as $k^{p}=$ $(-250.81,-15.91,-25.31), b^{p}=(-21,1.68,2.82)$, and $q_{e}^{p}=(0.40,1,0.28)$ for $\left(p^{1}, p^{2}, p^{3}\right)$. Tuning parameters of the VI controller is a challenging task that requires an expert to achieve good performance.

2) Proportional-Derivative Controller: In the experiments, the prosthetic knee torque of the PD controller is generated from

$$
u_{\mathrm{PD}}=-k_{p} e_{\mathrm{knee}}+k_{d} \dot{e}_{\mathrm{knee}}
$$

where $k_{p}$ and $k_{d}$ are design parameters. Since the effect of gravity is unknown to the PD controller, the control law of (41) yields steady state error on the prosthetic knee joint tracking. The tuning process of the PD controller is fairly straightforward. Increasing $k_{p}$ decreases the steady state error but may degrade the stability and increase overshoot. $k_{d}$ also affects the stability of the system and improves it if the value is small. The design parameters of the PD controller in both stance and nonstance phases are tuned as $k_{p}=100$ and $k_{d}=20$.

Remark 4: The design parameters of all proposed controllers in the experiments are listed in Table II. In the experiments, we initially use the same parameter values from simulation. We then tune the parameters within a small variation to achieve comfortable and sustainable walking. Table II confirms that the design parameters from the simulation can be directly applied to the prosthesis with minimal parameter tuning. The discrepancy between the parameter values in simulation and experiments stems from the hardware limitations and unmodeled dynamics between the model of Fig. 2(a) and the real human/prosthesis system. Thus, to achieve the best performance during experiment, design parameters need to be tuned with a small change from the simulation values.

\section{E. Experimental Results}

1) Treadmill Test: Fig. 9 demonstrates tracking performance of all model-based controllers over 2.5 min of walking for two consecutive steps along with their corresponding phase portraits. From Fig. 9, it can be seen that all proposed controllers as well as the RSC and RPC are able to track the prosthetic knee joint trajectories. This figure also shows that the system with the model-based controllers provides stable limit cycles. However, the AIC and ADC yield better knee angle tracking, while the RSC and RSAC provide the cleanest phase portraits, demonstrating greater consistency and robustness.

Fig. 9 also demonstrates tracking results and phase portraits of the prosthetic knee joint using the VI and PD controllers for 2.5 min of walking. This figure shows that all proposed modelbased controllers outperform both VI and PD controllers in 

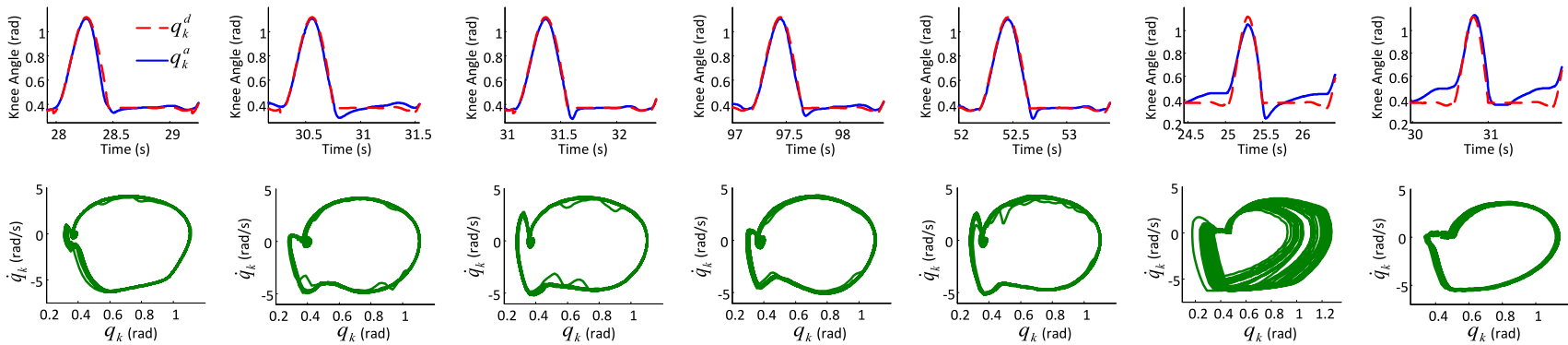

$\mathrm{ADC}$

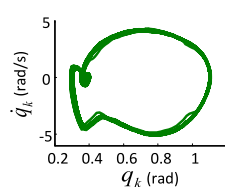

RSAC

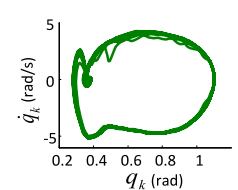

AIC

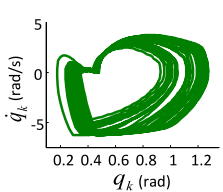

VI

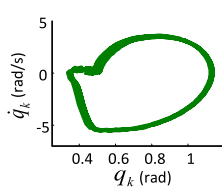

$\mathrm{PD}$

Fig. 9. Experimental tracking performance and phase portrait of the prosthetic knee joint for 2.5 min of walking on a treadmill using different controllers.

TABLE IV

Experimental Results of AMPRO3 Using the Three Proposed Approaches as Well as the RSC, RPC, VI, AND PD Controllers OVER 2.5 min OF WALKING ON A TREAdMILl. THE Best VALUe For EACH METRIC IS UNDERLined. $N_{p}$ Is the Number of TUning Parameters And $t_{t}$ Is the Tuning Time

\begin{tabular}{cccccccc}
\hline & RSC & RPC & ADC & RSAC & AIC & VI & PD \\
\hline \hline RMSE (rad) & 0.0389 & 0.0333 & 0.0229 & 0.0268 & $\underline{0.0218}$ & 0.0804 & 0.0778 \\
$E_{\max }(\mathrm{rad})$ & 0.1938 & 0.0910 & 0.0923 & 0.0962 & $\underline{0.0905}$ & 0.2068 & 0.1931 \\
$\tau_{\max }(\mathrm{N} . \mathrm{m})$ & 73 & 75 & 74 & $\underline{72}$ & 75 & 77 & 76 \\
$\mathrm{RMS}_{\tau}$ (N.m) & 17.77 & $\underline{15.90}$ & 20.11 & 17.93 & 19.88 & 17.70 & 16.30 \\
$\mathrm{~N}_{\mathrm{p}}$ & 24 & 18 & 28 & 36 & 28 & 9 & $\underline{4}$ \\
$\mathrm{t}_{\mathrm{t}}(\mathrm{min})$ & $\underline{5}$ & $\underline{5}$ & 7 & 7 & 10 & 30 & $\underline{5}$ \\
\hline
\end{tabular}

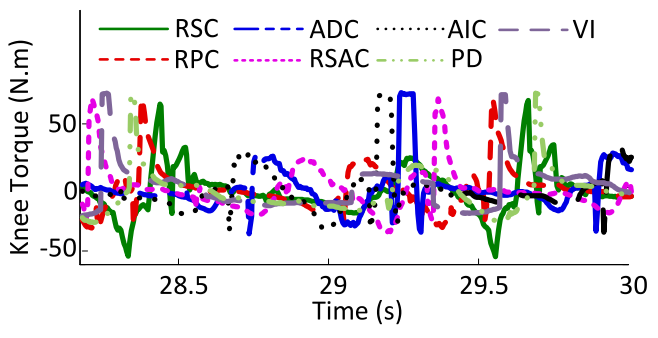

Fig. 10. Experimental prosthetic knee torque comparison between all proposed approaches as well as the RSC, RPC, VI, and PD controllers for a treadmill test.

tracking. Compared to the VI controller, the PD controller performs better regarding tracking performance and stability of the limit cycle.

Fig. 10 compares prosthetic knee torques for the proposed controllers as well as the RSC, RPC, VI, and PD controllers for a randomly selected time period $t \in[28,30] \mathrm{s}$. It can be seen that all the controllers generate similar absolute peak torque values at the end of the swing phase while a numerical comparison in Table IV shows that the RSAC uses the lowest maximum torque value.

Fig. 11 shows gait tiles of the human-prosthesis system walking in simulation and also experimentally using AMPRO3 with an able-bodied human test subject for all model-based controllers, visually demonstrating human-like walking.

2) Numerical Results for Treadmill Test: Table IV lists RMSE, maximum tracking error $E_{\max }$, maximum torque value $\tau_{\max }$, the RMS of the knee torque $\mathrm{RMS}_{\tau}$, number of the design parameters $N_{p}$, and tuning time of these parameters $t_{t}$ for AMPRO3 walking using the proposed controllers. This table also compares the results with the RSC, RPC, PD, and VI controllers over $2.5 \mathrm{~min}$ of walking. The best value for each metric is underlined in the table, showing that the best tracking performance and lowest $E_{\max }$ are achieved by the AIC, the RSAC has the lowest $\tau_{\max }$, and the RPC has the best $\mathrm{RMS}_{\tau}$. However, the RSAC provides the best compromise between tracking performance and torque. From inspection of Table IV, the tuning process of all five model-based controllers is easier than the VI controller in spite of having only nine parameters, which is less than the number of the design parameters of the proposed controllers. Table IV in general confirms that the proposed controllers outperform the VI and PD controllers regarding tracking performance and tuning, while providing a formal guarantee of stability and robustness in the presence of system uncertainties, unmodeled dynamics, and disturbances.

3) Outdoor Test (Setup): In addition to the treadmill test, an outdoor test is performed with AMPRO3 using the proposed controllers to verify their functionality in level ground and sloped walking. During the outdoor experiments, three able-bodied test subjects are asked to walk on level ground, uphill slopes, and downhill slopes for slope angles $3^{\circ}$ and $8^{\circ}$, while wearing the prosthesis. The subjects are a 25-year-old female (Subject \#1), a 28-year-old male (Subject \#2), and a 22-year-old male (Subject \#3). Subjects \#1 and \#2 performed the testing at California Institute of Technology (Caltech), and Subject \#3 carried it out at Georgia Institute of Technology. The subjects wearing AMPRO3 are shown in Fig. 1. It should be pointed out that the level ground walking test is done with all subjects and the sloped walking test is carried out only at Caltech with Subjects \#1 and \#2. Based on the building codes in the United States, the maximum slope of a ramp shall be approximately $5^{\circ}$ called normal slope [3]. The $3^{\circ}$ and $8^{\circ}$ slopes chosen in this paper are regarded as a mild slope and an extreme slope respectively.

Remark 5: It should be noted that during the outdoor tests, the design parameters are unchanged and are the same as used in the treadmill test (Table II). In other words, there is no 


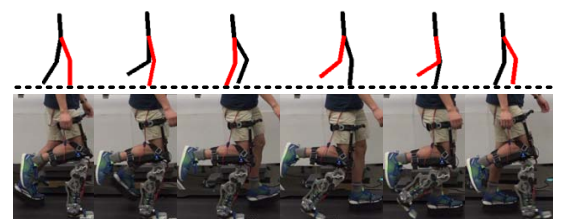

RSC

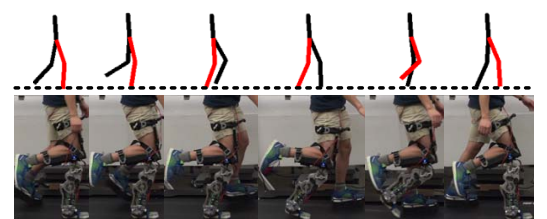

RPC

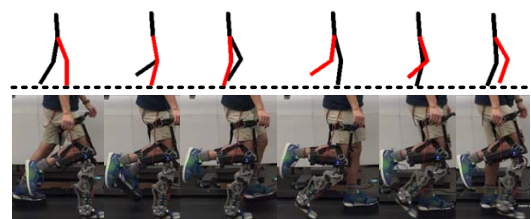

ADC

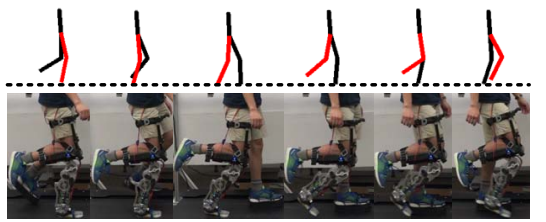

RSAC

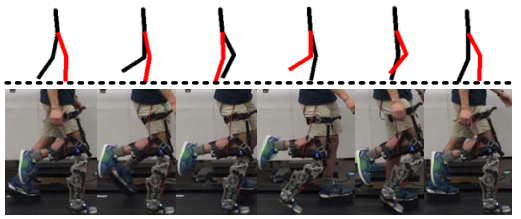

AIC

Fig. 11. Gait tiles of human-prosthesis walking in simulation and AMPRO3 walking with an able-bodied human test subject in a treadmill test over two steps using all proposed controllers.

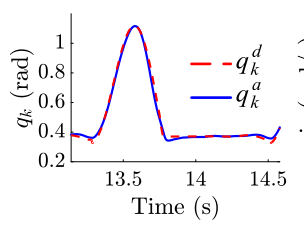

Subject \#1

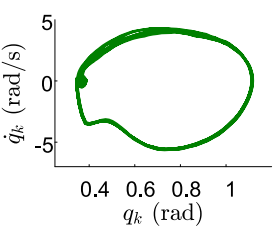

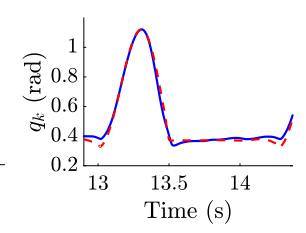

Subject \#2

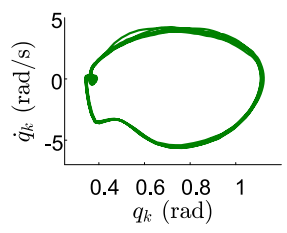

$4 k(\mathrm{rad})$
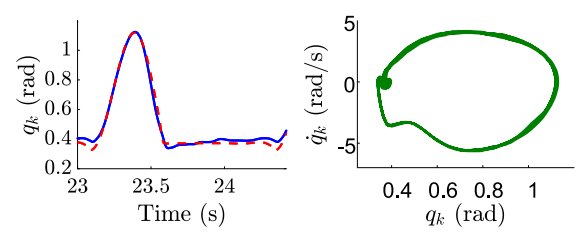

Subject \#3

Fig. 12. Tracking performance and phase portrait of the prosthetic knee for different subjects in outdoor level ground walking.

TABLE V

PEQ for Three Able-Bodied SubJects in the Outdoor Test. The QUESTIONNAIRE IS CATEGORIZED IN FOUR DIFFERENT GROUPS AS Group \#1: Device SATISFACtion, Group \#2: Bodily SEnSATION, Group \#3: ABILITY TO MOVE, AND Group \#4: TrAining Satisfaction. Each Subject (Subject \#1, Subject \#2, and Subject \#3) Rates Her/His Satisfaction on a Scale From 1 to 10 (1: Fully UNSATISFIED AND 10: FUlly SATISFIED) IN DIFFERENT GROUPS

\begin{tabular}{cccc}
\hline Question & Scale & Question & Scale \\
\hline \hline fitting $^{1}$ & $(6,9,5)$ & sound $^{1}$ & $(5,10,3)$ \\
weight $^{1}$ & $(4,8,7)$ & pain in connected leg & $(4,8,2)$ \\
comfort while standing $^{1}$ & $(6,5,8)$ & pain in other leg & $(7,9,8)$ \\
balance while using $^{1}$ & $(7,7,5)$ & back pain $^{2}$ & $(8,10,10)$ \\
socket comfortability $^{1}$ & $(4,8,3)$ & ability to walk $^{3}$ & $(6,8,7)$ \\
ease of wearing & $(8,7,6)$ & training to walk $^{4}$ & $(9,10,9)$ \\
\hline average of Group \#1 & $(5.7,7.7,5.3)$ \\
average of Group \#2 & $(6.3,9,6.6)$ \\
average of Group \#3 & $(6,7,8)$ & \\
average of Group \#4 & $(9,10,9)$ \\
\hline
\end{tabular}

need to adjust the controller parameters for different subjects, or for level and sloped walking. This shows that controllers are robust enough to deal with different users and different walking surfaces.

After testing, the subjects are asked to fill out a questionnaire to evaluate their satisfaction with the device: the prosthesis evaluation questionnaire (PEQ). The PEQ is categorized in four different groups (Group \#1: Device satisfaction, Group \#2: Bodily sensation, Group \#3: Ability to move, and Group \#4: Training satisfaction) to evaluate each subject's satisfaction and to indicate the functionality of prosthesis walking/quality of life. This questionnaire is a modified version of the PEQ [43] based on our experiments with AMPRO3 walking and healthy test subjects. The results of the questionnaire are given in Table $\mathrm{V}$. The subjects also provided written

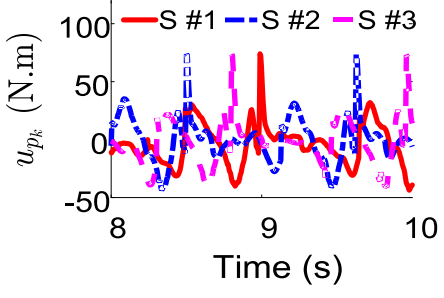

Fig. 13. Torque of the prosthetic knee for different subjects in outdoor level ground walking ( $\mathrm{S} \# 1$ : first subject, $\mathrm{S} \# 2$ : second subject, and $\mathrm{S} \# 3$ : third subject).

feedback on each component of the survey. While the subjects are able to put the device on independently after some practice, they comment on the need to have the straps of the iWalk adapter be very tight in order to feel confident in having a secure connection with the device. This leads to discomfort in the connected leg due to the tightness and rubbing of the straps when in contact with skin. However, the subjects do not experience much pain in their other leg or their back; the only noted pain is due to fatigue from extra work to compensate for the weight of the device and to ensure balance. One subject comments on the slight struggle to balance on the device with the extra weight and the shoe lift. Another subject mentions she had to control her balance in the frontal plane since the prosthesis is not actuated in this direction, but balance in the sagittal plane is satisfactory. The sound of the device does not bother one subject, but the other subjects comment on how this sound would be bothersome for daily use and a silent device would be preferred.

The subjects are moderately satisfied with their ability to walk with the device, although it takes practice to become accustomed to the extra mass, requiring extra attention to lift 

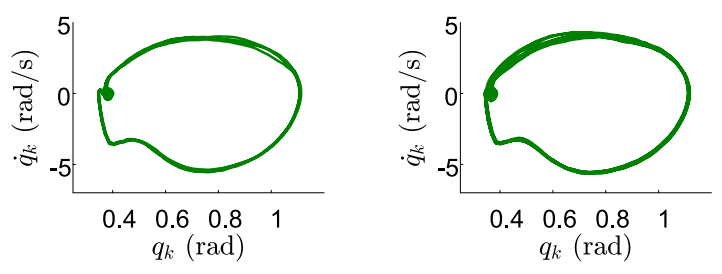

(a)

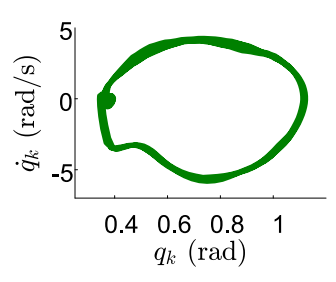

(c)

Subject \#1

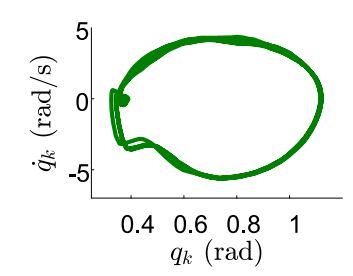

Subject \#2
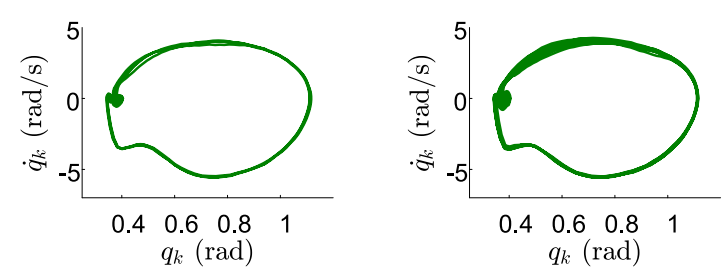

(b)
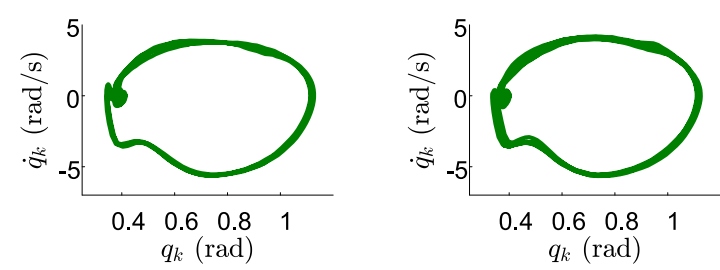

(d)

Subject \#1

Subject \#2

Fig. 14. Phase portraits of the prosthesis knee for different subjects in outdoor sloped walking of $3^{\circ}$ and $8^{\circ}$. (a) Downslope walking 3. (b) Upslope walking 3. (c) Downslope walking 8. (d) Upslope walking 8.

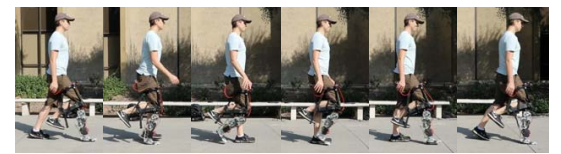

(a)

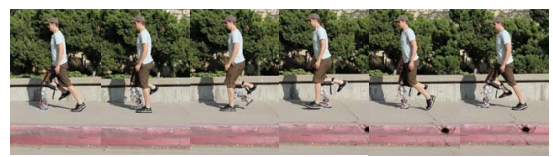

(b)

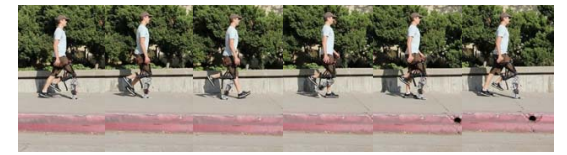

(c)

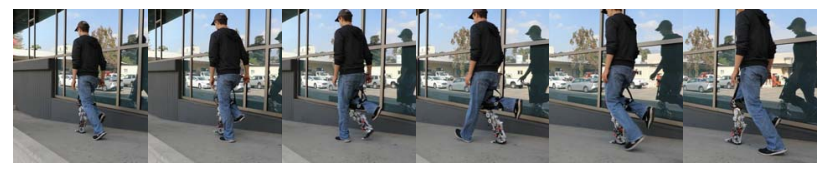

(d)

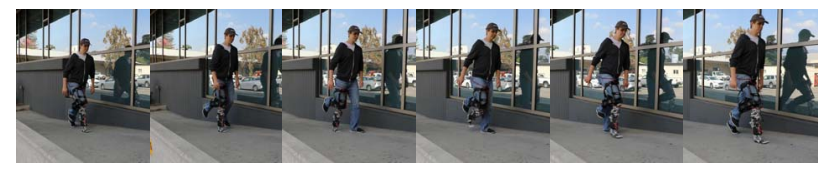

(e)

Fig. 15. Gait tiles of outdoor AMPRO3 walking on level ground, uphill slopes, and downhill slopes of $3^{\circ}$ and $8^{\circ}$. (a) Level ground walking. (b) Downhill walking 3. (c) Uphill walking 3. (d) Downhill walking 8. (e) Uphill walking 8.

the device to prevent foot scuffing. This foot scuffing risk increases for walking up slopes, but is the only noted additional challenge for this terrain. For walking down slopes, one subject commented on the need to take smaller steps in order to feel stable due to the small, hard plastic foot which does not provide much grip on the ground or sense of security. Another subject notes that the device would sometimes not initiate a step when going down a slope. On the slopes, the ankle conforms nicely to the angle of the slope. All of the subjects are very satisfied with their training of the device and comment on the adequate time and support to adjust to the device and rest as needed.

4) Outdoor Test (Results): During testing, data for the calculated desired position and velocity of the knee as well as the actual position and velocity of the knee are recorded, as measured by the on-board encoder. The actual knee torque is also calculated based on the current measured by the motor controllers. The subjects walk on flat ground for stretches of about $10 \mathrm{~m}$. Fig. 12 shows tracking performance, phase portrait, and torque of the prosthetic knee for different subjects in outdoor level ground walking. It is observed that the proposed controller can accurately track the desired knee position and provide convergence of the knee trajectory to a stable periodic orbit for all three subjects. According to Fig. 13, it can also be seen that the knee torques for different subjects have relatively similar magnitudes compared to the data from treadmill tests.

Subjects \#1 and \#2 walk up and down slopes of $3^{\circ}$ and $8^{\circ}$, measured by a digital angle meter. These slopes are about 10.4 and $5.6 \mathrm{~m}$ long, respectively. All of the tests are performed outdoors on concrete sidewalks. At least five trials are done for each terrain type consisting of 10 strides or more. Fig. 14 illustrates phase portraits of the prosthetic knee for different subjects in outdoor sloped walking for $3^{\circ}$ and $8^{\circ}$. It is seen that the controller can provide stable limit cycles for the knee trajectories for different subjects walking on the mild and extreme slopes. This implies that the proposed controller is able to provide stable walking for uphill and downhill walking. Fig. 15 shows gait tiles of outdoor AMPRO3 walking on level ground, uphill slopes, and downhill slopes for $3^{\circ}$ and $8^{\circ}$. This figure shows that the proposed controller can provide qualitatively human-like walking for subjects on level ground, and both uphill slopes and downhill slopes of $3^{\circ}$ and $8^{\circ}$. Based on our experiments, sloped walking does not require higher torque at the knee than outdoor level ground and treadmill walking. 
To visualize the performance of the proposed controllers, AMPRO3 walking results using the proposed controllers in simulation and experiments (treadmill and outdoor tests) can be seen in a video available at [25].

\section{DISCUSSION AND CONCLUSION}

The VI and PD controllers are widely used for prosthetic leg control, wherein they have the advantage that they can be implemented in a model independent fashion. However, they are not amenable to formal stability analysis and do not provide proper tracking performance due to the presence of parameter uncertainties, unmodeled dynamics, and disturbances. This motivated the results presented in this paper.

In this paper, we designed and experimentally tested three different controllers to control a transfemoral prosthesis, addressing the above issues. The stability of all three controllers was proven using the Lyapunov methods. This provides the first steps toward model-based adaptive and robust ADCs to mitigate the disadvantages of the VI and PD controllers in that they give formal stability guarantees and, as shown in this paper, improved performance. The proposed controllers were first verified on a walking biped platform and then successfully implemented on AMPRO3.

Simulation results showed that all proposed controllers provided good prosthetic knee tracking performance and humanlike walking. Their results were compared against each other and also with RSC and RPC results. The RSC provided the best robustness and stability in the presence of disturbances, the AIC provided the best tracking performance, and the RSAC provided the best compromise between these objectives.

All proposed model-based controllers were experimentally verified on AMPRO3 using able-bodied test subjects on a treadmill and compared with the RSC, RPC, VI, and PD controllers. Treadmill results showed that all newly proposed controllers outperformed both VI and PD controllers in tracking and parameter tuning time. In addition to the treadmill test, an outdoor test was performed using AMPRO3 with three ablebodied test subjects walking on level ground, uphill slopes, and downhill slopes of $3^{\circ}$ and $8^{\circ}$. The outdoor tests revealed that the proposed approaches were able to provide human-like and stable walking for AMPRO3 on different uneven surfaces.

Future work would aim to consider the following tasks.

1) In this paper, the robustness test was only performed in simulation for the biped model. However, additional experiments can be performed to quantify robustness to unexpected pushes and obstacles using the proposed controllers.

2) The effect of GRF was not considered, so an observer/controller combination can be designed for the biped platform in simulation, and then experimentally implemented on AMPRO3 to provide GRF estimationbased control.

3) This paper presented different model-based controllers for AMPRO3 while the ankle joint was assumed to be passive. However, a powered ankle is able to provide positive net work and power during the stance phase while walking at moderate to fast walking speeds [44], [45]. Thus, modifying the proposed controllers to include both knee and ankle could reduce the metabolic energy cost of walking, in turn enhancing the quality of gait.

4) This paper validated the soundness of model-based controllers for walking on level ground and ramps. However, stair ascent and descent remain interesting problems that could be addressed in future work with extensions of the proposed controllers. These controllers along with stable human-like gait generation could provide a stair ascent and descent control system for a powered knee and ankle prosthesis to further empower amputees in their daily life.

\section{ACKNOWLEDGMENT}

The authors are indebted to J. Reher for his help on the outdoor experimental tests. They would like to thank E. Ambrose for designing and building the prosthesis device AMPRO3. They would also like to thank the Editor and reviewers for their helpful comments and suggestions. This research was approved by the Georgia Institute of Technology and the California Institute of Technology Institutional Review Boards, protocol numbers H15255 and 16-0693, for testing with humans.

\section{REFERENCES}

[1] T. Dillingham, L. E. Pezzin, and E. J. MacKenzie, "Limb amputation and limb deficiency: Epidemiology and recent trends in the United States," Southern Med. J., vol. 95, no. 8, pp. 875-884, 2002.

[2] Z. W. Lui, M. I. Awad, A. Abouhossein, A. A. Dehghani-Sanij, and N. Messenger, "Virtual prototyping of a semi-active transfemoral prosthetic leg," in Proc. Inst. Mech. Eng. H J. Eng. Med., vol. 229, no. 5, pp. 350-361, 2015.

[3] F. Sup, H. A. Varol, and M. Goldfarb, "Upslope walking with a powered knee and ankle prosthesis: Initial results with an amputee subject," IEEE Trans. Neural Syst. Rehabil. Eng., vol. 19, no. 1, pp. 71-78, Feb. 2011.

[4] D. A. Winter, The Biomechanics and Motor Control of Human Gait: Normal, Elderly, and Pathological. Waterloo, ON, Canada: Univ. Waterloo Press, 1991.

[5] B. E. Lawson, H. A. Varol, and M. Goldfarb, "Standing stability enhancement with an intelligent powered transfemoral prosthesis," IEEE Trans. Biomed. Eng., vol. 58, no. 9, pp. 2617-2624, Sep. 2011.

[6] A. M. Simon et al., "Delaying ambulation mode transition decisions improves accuracy of a flexible control system for powered knee-ankle prosthesis," IEEE Trans. Neural Syst. Rehabil. Eng., vol. 25, no. 8, pp. 1164-1171, Aug. 2017.

[7] F. Sup, A. Bohara, and M. Goldfarb, "Design and control of a powered transfemoral prosthesis," Int. J. Robot. Res., vol. 27, no. 2, pp. 263-273, 2008.

[8] N. P. Fey, A. M. Simon, A. J. Young, and L. J. Hargrove, "Controlling knee swing initiation and ankle plantarflexion with an active prosthesis on level and inclined surfaces at variable walking speeds," IEEE $J$. Transl. Eng. Health Med., vol. 2, pp. 1-12, 2014.

[9] C. G. Atkeson and S. Schaal, "Robot learning from demonstration," in Proc. Int. Conf. Mach. Learn., vol. 97, 1997, pp. 12-20.

[10] H. Zhao, J. Horn, J. Reher, V. Paredes, and A. D. Ames, "First steps toward translating robotic walking to prostheses: A nonlinear optimization based control approach," Auton. Robots, vol. 19, no. 1, pp. 1-18, 2011.

[11] H. Zhao, J. Horn, J. Reher, V. Paredes, and A. D. Ames, "Multicontact locomotion on transfemoral prostheses via hybrid system models and optimization-based control," IEEE Trans. Autom. Sci. Eng., vol. 13, no. 2, pp. 502-513, Apr. 2016.

[12] Y. Wen, J. Si, X. Gao, S. Huang, and H. H. Huang, "A new powered lower limb prosthesis control framework based on adaptive dynamic programming," IEEE Trans. Neural Netw. Learn. Syst., vol. 28, no. 9, pp. 2215-2220, Sep. 2017. 
[13] F. Ferraguti, C. Secchi, and C. Fantuzzi, "A tank-based approach to impedance control with variable stiffness," in Proc. IEEE Int. Conf. Robot. Autom., Karlsruhe, Germany, 2013, pp. 4948-4953.

[14] K. Kronander and A. Billard, "Stability considerations for variable impedance control," IEEE Trans. Robot., vol. 32, no. 5, pp. 1298-1305, Oct. 2016.

[15] W. He et al., "Model identification and control design for a humanoid robot," IEEE Trans. Syst., Man, Cybern., Syst., vol. 47, no. 1, pp. 45-57, Jan. 2017.

[16] M. Van, M. Mavrovouniotis, and S. S. Ge, "An adaptive backstepping nonsingular fast terminal sliding mode control for robust fault tolerant control of robot manipulators," IEEE Trans. Syst., Man, Cybern., Syst., to be published. doi: 10.1109/TSMC.2017.2782246.

[17] J. Yao and W. Deng, "Active disturbance rejection adaptive control of uncertain nonlinear systems: Theory and application," Nonlin. Dyn., vol. 89, no. 3, pp. 1611-1624, Aug. 2017.

[18] W. Deng and J. Yao, "Adaptive integral robust control and application to electromechanical servo systems," ISA Trans., vol. 67, pp. 256-265, Mar. 2017.

[19] V. Azimi, T. T. Nguyen, M. Sharifi, S. A. Fakoorian, and D. Simon, "Robust ground reaction force estimation and control of lower-limb prostheses: Theory and simulation," IEEE Trans. Syst., Man, Cybern., Syst., to be published. doi: 10.1109/TSMC.2018.2836913.

[20] V. Azimi et al., "Robust control of a powered transfemoral prosthesis device with experimental verification," in Proc. Amer. Control Conf., Seattle, WA, USA, 2017, pp. 517-522.

[21] H. Ma et al., "Neural-network-based distributed adaptive robust control for a class of nonlinear multiagent systems with time delays and external noises," IEEE Trans. Syst., Man, Cybern., Syst., vol. 46, no. 6, pp. 750-758, Jun. 2016.

[22] C. Sun, W. He, W. Ge, and C. Chang, "Adaptive neural network control of biped robots," IEEE Trans. Syst., Man, Cybern., Syst., vol. 47, no. 2, pp. 315-326, Feb. 2017.

[23] L. Ding et al., "Adaptive neural network-based tracking control for fullstate constrained wheeled mobile robotic system," IEEE Trans. Syst., Man, Cybern., Syst., vol. 47, no. 8, pp. 2410-2419, Aug. 2017.

[24] R. Cui, C. Yang, Y. Li, and S. Sharma, "Adaptive neural network control of AUVs with control input nonlinearities using reinforcement learning," IEEE Trans. Syst., Man, Cybern., Syst., vol. 47, no. 6, pp. 1019-1029, Jun. 2017

[25] V. Azimi. Experimental and Simulation Results of AMPRO3 Walking. Accessed: Nov. 20, 2018. [Online]. Available: https://youtu.be/7tNctFvII5o

[26] E. R. Westervelt, J. W. Grizzle, C. Chevallereau, J. H. Choi, and B. Morris, Feedback Control of Dynamic Bipedal Robot Locomotion. Boca Raton, FL, USA: CRC Press, 2007.

[27] A. D. Ames, "First steps toward automatically generating bipedal robotic walking from human data," in Robot Motion and Control 2011 (Lecture Notes in Control and Information Sciences), vol. 422. K. Kozłowski, Ed. London, U.K.: Springer, 2012.

[28] M. Spong, S. Hutchinson, and M. Vidyasagar, Robot Modeling and Control. Hoboken, NJ, USA: Wiley, 2005.

[29] Y. Hurmuzlu and D. B. Marghitu, "Rigid body collisions of planar kinematic chains with multiple contact points," Int. J. Robot. Res., vol. 13, no. 1, pp. 82-92, 1994.

[30] E. R. Westervelt, J. W. Grizzle, and D. E. Koditschek, "Hybrid zero dynamics of planar biped walkers," IEEE Trans. Autom. Control, vol. 48, no. 1, pp. 42-56, Jan. 2003.

[31] A. D. Ames, "Human-inspired control of bipedal walking robots," IEEE Trans. Autom. Control, vol. 59, no. 5, pp. 1115-1130, May 2014.

[32] H. Zhao, "From bipedal locomotion to prosthetic walking: A hybrid system and nonlinear control approach," Ph.D. dissertation, Mech. Eng., Georgia Inst. Technol., Atlanta, GA, USA, 2016.

[33] H. Zhao, M. Powell, and A. D. Ames, "Human-inspired motion primitives and transitions for bipedal robotic locomotion in diverse terrain," Opt. Control Appl. Methods, vol. 35, no. 6, pp. 730-755, 2013.

[34] A. D. Ames, K. Galloway, K. Sreenath, and J. W. Grizzle, "Rapidly exponentially stabilizing control Lyapunov functions and hybrid zero dynamics," IEEE Trans. Autom. Control, vol. 59, no. 4, pp. 876-891, Apr. 2014

[35] J.-J. E. Slotine and W. Li, "Adaptive manipulator control: A study case," IEEE Trans. Autom. Control, vol. 33, no. 11, pp. 995-1003, Nov. 1988.

[36] J.-J. E. Slotine and W. Li, Applied Nonlinear Control. Englewood Cliffs, NJ, USA: Prentice-Hall, 1991.

[37] J.-J. E. Slotine and J. A. Coetsee, "Adaptive sliding controller synthesis for non-linear systems," Int. J. Control, vol. 43, no. 6, pp. 1631-1651, 1984.
[38] V. Azimi, D. Simon, and H. Richter, "Stable robust adaptive impedance control of a prosthetic leg," in Proc. ASME Dyn. Syst. Control Conf., Columbus, OH, USA, 2015, Art. no. V001T09A003. doi: 10.1115/DSCC2015-9794.

[39] V. Azimi, D. Simon, H. Richter, and S. A. Fakoorian, "Robust composite adaptive transfemoral prosthesis control with non-scalar boundary layer trajectories," in Proc. Amer. Control Conf., Boston, MA, USA, 2016, pp. 3002-3007.

[40] H. Zhao, E. Ambrose, and A. D. Ames, "Preliminary results on energy efficient 3D prosthetic walking with a powered compliant transfemoral prosthesis," in Proc. IEEE Int. Conf. Robot. Autom., Singapore, 2017, pp. 1140-1147.

[41] P. W. Duncan, D. K. Weiner, J. Chandler, and S. Studenski, "Functional reach: A new clinical measure of balance," J. Gerontol., vol. 45, no. 6 , pp. M192-M197, Nov. 1990.

[42] D. Brooks, J. Parsons, J. P. Hunter, M. Devlin, and J. Walker, "The 2-minute walk test as a measure of functional improvement in persons with lower limb amputation," Archives Phys. Med. Rehabil., vol. 82, no. 10, pp. 1478-1483, 2001

[43] Prosthesis Evaluation Questionnaire, Prosthetics Res. Study, Seattle, WA, USA, 1998.

[44] A. Hansen, D. Childress, S. Miff, S. Gard, and K. Mesplay, "The human ankle during walking: Implication for the design of biomimetric ankle prosthesis," J. Biomech., vol. 37, no. 10, pp. 1467-1474, 2004.

[45] S. K. Au, J. Weber, and H. Herr, "Powered ankle-foot prosthesis improves walking metabolic economy," IEEE Trans. Robot., vol. 25, no. 1, pp. 51-66, Feb. 2009.

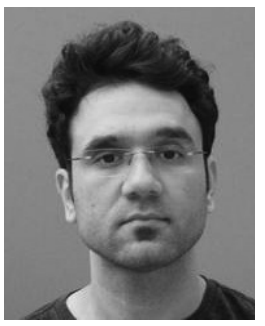

Vahid Azimi is currently pursuing the Ph.D. degree in electrical engineering with the Georgia Institute of Technology, Atlanta, GA, USA

He joined the AMBER Laboratory, Georgia Institute of Technology in 2016, where his research focused on prosthetic devices and walking bipeds. His current research interests include nonlinear control, robotics, optimization, and multiobjective quadratic program-based robust adaptive control of fully actuated/underactuated robotic systems in the presence of modeling uncertainty and unknown robot-environment interaction.

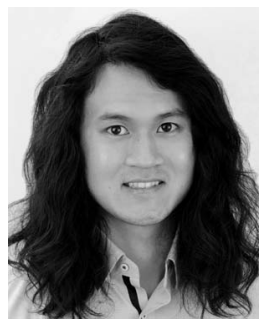

Tony Shu received the B.S. degree in materials science and engineering from the Georgia Institute of Technology, Atlanta, GA, USA, in 2016. He is currently pursuing the graduation degree in media arts and sciences with the Massachusetts Institute of Technology, Cambridge, MA, USA.

His current research interest includes leverages biomechanical models to enable intuitive control of external devices through native body signals.

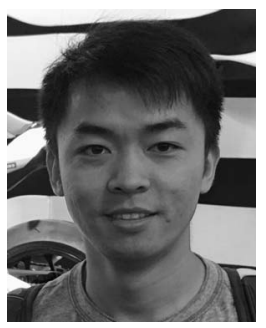

Huihua Zhao received the B.S. degree in mechanical engineering from the University of Science and Technology of China, Hefei, China, in 2010 and the $\mathrm{Ph} . \mathrm{D}$. degree in mechanical engineering from the Georgia Institute of Technology, Atlanta, GA, USA, in 2016.

He is currently a Research Scientist with Toyota Research Institute, San Francisco, CA, USA. His current research interests include motion planning, trajectory optimization, and optimal control of robotic systems. 


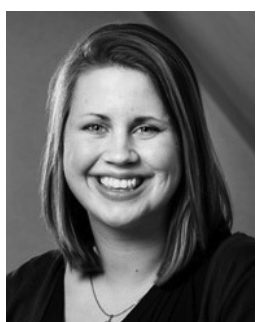

Rachel Gehlhar received the B.S. degree in mechanical engineering from the University of St. Thomas, Saint Paul, MN, USA, in 2016 and the M.S. degree in mechanical engineering from the California Institute of Technology, Pasadena, CA, USA, in 2018, where she is currently pursuing the Ph.D. degree in mechanical engineering.

Her research focuses on developing formal nonlinear control methods for powered prostheses to achieve safe and stable walking for amputees. She is specifically pursuing synergizing human sensory methods with powered prostheses to empower prostheses to be more autonomous, for the purpose of both robustness and adaptability to the individual human user.

Ms. Gehlhar was a recipient of the Graduate Research Fellowship from the National Science Foundation in 2017.

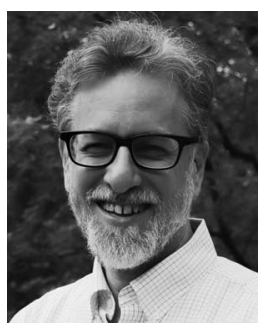

Dan Simon received the B.S. degree in electrical engineering from Arizona State University, Tempe, AZ, USA, in 1983, the M.S. degree in electrical engineering from the University of Washington, Seattle, WA, USA, in 1987, and the Ph.D. degree in electrical engineering from Syracuse University, Syracuse, NY, USA, in 1991.

He has had 14 years of industrial experience in several engineering fields, including aerospace, automotive, biomedical, process control, and software. He joined Cleveland State University, Cleveland, OH, USA, in 1999, where he is currently a Professor with the Electrical Engineering and Computer Science Department, and the Associate Vice President for Research. He is the Director of the Embedded Control Systems Research Laboratory, Colorado State University, Fort Collins, CO, USA, which has received funding from NASA, NSF, Cleveland Clinic, and industry. He has about 200 peer-reviewed publications. He has authored the book entitled Optimal State Estimation (Wiley, 2006), Evolutionary Optimization Algorithms (Wiley, 2013), and Evolutionary Computation With Biogeography-Based Optimization (Wiley, 2017). His publications, teaching materials, and research-related software can be download from his Web site at http://academic.csuohio.edu/simond/. His current research interests include evolutionary algorithms, computer intelligence, and control theory.

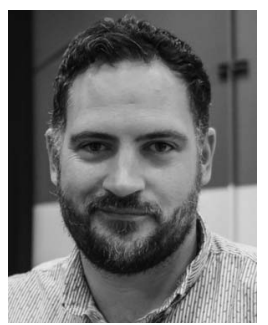

Aaron D. Ames received the B.S. degree in mechanical engineering and the B.A. degree in mathematics from the University of St. Thomas, Saint Paul, MN, USA, in 2001, and the M.A. degree in mathematics and the Ph.D. degree in electrical engineering and computer sciences from the University of California at Berkeley (UC Berkeley), Berkeley, CA, USA, in 2006.

He was an Associate Professor of Mechanical Engineering and Electrical and Computer Engineering with the Georgia Institute of Technology, Atlanta, GA, USA. He served as a Post-Doctoral Scholar of Control and Dynamical Systems with the California Institute of Technology, Pasadena, CA, USA, from 2006 to 2008, where he is the Bren Professor of Mechanical and Civil Engineering and Control and Dynamical Systems. He began his faculty career with Texas A\&M University, College Station, TX, USA, in 2008. His current research interests include robotics, nonlinear control, and hybrid systems, with a special focus on applications to bipedal robotic walking both formally and through experimental validation. His lab designs, builds, and tests novel bipedal robots, humanoids and prostheses with the goal of achieving human-like bipedal robotic locomotion and translating these capabilities to robotic assistive devices. The application of these ideas ranges from increased autonomy in robots to improve the locomotion capabilities of the mobility impaired.

Dr. Ames was a recipient of the 2005 Leon O. Chua Award for achievement in nonlinear science and the 2006 Bernard Friedman Memorial Prize in Applied Mathematics from UC Berkeley, the NSF CAREER Award in 2010, and the 2015 Donald P. Eckman Award. 